\title{
PATRONES DE DISTRIBUCIÓN, ABUNDANCIA Y RIQUEZA DE ESPECIES DE LA AVIFAUNA TERRESTRE DE LA ISLA DE LA PALMA (ISLAS CANARIAS)
}

\author{
L. M. Carrascal ${ }^{1}$, D. Palomino ${ }^{2} \&$ V. Polo ${ }^{3}$
}

\begin{abstract}
RESUMEN
Se estiman los niveles poblacionales, las densidades ecológicas, los principales patrones de preferencia de hábitat, las posibles tendencias demográficas habidas en los últimos 25 y 15 años, y el estatus de conservación actual de las especies de aves terrestres diurnas reproductoras en la isla de La Palma. Los datos fueron obtenidos durante el periodo reproductor de 2007, utilizando transectos lineales. En total se efectuaron 437 transectos de $0,5 \mathrm{~km}$ repartidos por toda la isla atendiendo a sus formaciones ambientales, situación altitudinal, y posición geográfica.

Mediante curvas acumuladas del número de especies se explora la variación en la riqueza de especies. El ambiente con menor biodiversidad aviar es, con diferencia, el matorral altimontano. Por el contrario, los medios con mayor riqueza de especies son las medianías dedicadas a la agricultura y con casas dispersas. La variación local de la riqueza de aves (especies por transecto de $0,5 \mathrm{~km}$ ) en la isla de La Palma tiene una importante componente geográfica (aumento del sur al norte y del oeste al este, con valores máximos a altitudes medias), asociándose además con el desarrollo de la vegetación especialmente en los estratos arbustivos y herbáceo, y estando negativamente influida por el desarrollo de la agricultura pero no por el urbanismo. La densidad total de aves alcanza sus máximos valores en la laurisilva $\left(657 \mathrm{aves} / \mathrm{km}^{2}\right)$, fayal-brezal $\left(630 \mathrm{aves} / \mathrm{km}^{2}\right)$ y la transición pinar-laurisilva $\left(509\right.$ aves $\left./ \mathrm{km}^{2}\right)$, mientras que los matorrales de alta montaña $\left(153\right.$ aves $\left./ \mathrm{km}^{2}\right)$ y las lavas recientes $\left(58\right.$ aves $\left./ \mathrm{km}^{2}\right)$ mantienen avifaunas poco densas. Las especies que tienen niveles poblacionales más críticos en la isla son Falco [peregrinus] pelegrinoides, Burhinus oedicnemus distinctus, Upupa epops, Carduelis carduelis, Miliaria calandra y Petronia petronia, habiendo manifestado las últimas cinco especies importantes regresiones en los últimos 15-25 años.
\end{abstract}

Palabras clave: Aves, Islas Canarias, La Palma, riqueza de especies, densidad, estructura del hábitat, tamaños de población, estatus de conservación.

\section{SUMMARY}

\section{Patterns of distribution, abundance and species richness of terrestrial bird fauna in La Palma (Canary islands)}

We estimate the population size and density, habitat preferences, habitat breadth and probable population trends in the last 25-15 years of the diurnal terrestrial bird species breeding in La Palma island (Canary islands). Data were obtained in a large extensive

1 Dept. Biodiversidad y Biología Evolutiva, Museo Nacional de Ciencias Naturales, CSIC. C/ José Gutiérrez Abascal 2, 28006 Madrid, Spain. Email: mcnc152@mncn.csic.es

2 Área de Estudio y Seguimiento de Aves, SEO/BirdLife. C/ Melquiades Biencinto 34, 28053 Madrid, Spain.

3 Grupo de Conservación y Biodiversidad, Departamento de Biología y Geología, Universidad Rey Juan Carlos, E-28933 Móstoles (Madrid), Spain. 
census program carried out during the 2007 nesting period using line transects that allowed detectability estimations.

We also explored patterns of species richness both at inter-habitat and local scale. Local species richness (species per $0.5 \mathrm{~km}$ transects) showed an important geographical component, increasing from south to north, from west to east, and reaching maximum values at altitudes around $600-1,000 \mathrm{~m}$ a.s.l. It was also positively related to vegetation development (specially in the herbaceous and shrub layer), and negatively associated with urbanization and agriculture. Total bird density reached the highest figures in the 'monteverde' (laurel and heath forests ca. 650 aves $/ \mathrm{km}^{2}$ ), and in the transition pinewoods-laurel forests $\left(509\right.$ aves $\left./ \mathrm{km}^{2}\right)$, while the lowest figures were recorded in high altitude shrublands $\left(153\right.$ aves $\left./ \mathrm{km}^{2}\right)$ and recent lava fields $\left(58\right.$ aves $\left./ \mathrm{km}^{2}\right)$. The bird species with lower population sizes are Falco [peregrinus] pelegrinoides, Burhinus oedicnemus distinctus, Upupa epops, Carduelis carduelis, Miliaria calandra and Petronia petronia, while the last five species have undergone more negative population trends in the last 15-25 years .

Key words: Birds, Canary Islands, La Palma, species richness, density, habitat structure, population sizes, conservation status.

\section{Introduccion}

La avifauna de la isla de La Palma se encuentra entre una de las más desconocidas del archipiélago Canario. A ello ha contribuido su aislamiento geográfico y la ausencia de instituciones académicas relacionadas con la investigación en biodiversidad. La mayoría de la información disponible se restringe a resúmenes de excursiones ornitológicas, o citas de observaciones concretas, que aunque de un incalculable valor faunístico, no permiten establecer patrones claros de biodiversidad o rareza ecológica de la avifauna. Los informes técnicos, aun presentando mucho mayor rigor, se remiten a unas pocas especies y tienen una difusión muy restringida, lo que limita mucho su utilidad y transmisión del conocimiento. Es más, principalmente se refieren a unas pocas especies emblemáticas o raras, dejando de lado a la gran mayoría de la biodiversidad aviar de la isla. No obstante, la excelente revisión de Martín y Lorenzo (2001) y el Atlas de las Aves Nidificantes en el Archipiélago Canario (Lorenzo, 2007) han supuesto un avance considerable en el conocimiento de la avifauna de La Palma, al sintetizar toda esa información dispersa en distintas fuentes de difícil acceso. Por otro lado, las escasas referencias de literatura científica sobre aves de La Palma disponibles en bases internacionales (sólo 9 incluidas en el ISI World of Knowledge a Marzo de 2008 para el periodo 19782008), no se refieren directamente a aspectos demográficos o de biodiversidad, con la excepción de Packert et al. (2006) y Packert (2006) que describen una nueva subespecie de ave para la isla (Regulus regulus ellenthalerae), Kvist et al. (2005) y Dietzen et al. (2008) que analizan el complejo canario de
Cyanistes teneriffae, Baker et al. (1990) que analizan la divergencia genética de poblaciones periféricas insulares de Fringilla coelebs, y Carrascal y Palomino (2002) y Donazar et al. (2005) que definen grandes patrones biogeográficos y de extinción de especies en el archipiélago Canario en general.

La isla de La Palma tiene una riqueza ornitológica actual media en el contexto canario, la cual puede ser explicada claramente atendiendo a la diversidad de ambientes que incluye (derivado de su elevado gradiente altitudinal), su aislamiento geográfico (distancia al continente) y su superficie (Carrascal y Palomino, 2002). De las 35 especies de aves terrestres reproductoras en la isla, no se tiene conocimiento cuantitativo preciso de sus patrones de abundancia, preferencias de hábitat y efectos de los cambios del uso del suelo sobre sus efectivos (si se exceptúa el caso de las palomas de laurisilva). Esta información sería de valor para establecer objetivamente la rareza de su avifauna, de manera que pudiera ayudar a definir objetivamente sus niveles de amenaza en el establecimiento de listas rojas, y estableciesen medidas cuantitativas de niveles demográficos que pudiesen ser utilizadas en el futuro para poder aplicar los criterios de la UICN a nivel regional (Gärdenfors, 2001; Gärdenfors et al., 2001).

Los objetivos de este trabajo son los siguientes:

- estimar la densidad de las distintas especies de aves terrestres en las principales formaciones ambientales de la isla, describiendo sus principales preferencias de hábitat

- estimar el tamaño de sus poblaciones en toda la isla, que sirva como referente cuantitativo frente 
al cual se puedan comparar los resultados obtenidos en el futuro siguiendo la misma o similar metodología, para poder establecer tendencias demográficas objetivas necesarias al aplicar criterios de catalogación UICN,

- establecer los principales patrones globales de biodiversidad aviar en la isla.

\section{Material y métodos}

El estudio se ha llevado a cabo en la isla de La Palma (708 km²; Islas Canarias). Los muestreos se llevaron a cabo durante las tres últimas semanas del mes de Abril y primera semana de Mayo de 2007, fechas que corresponden con el periodo reproductor de las aves en esta isla (Martín y Lorenzo, 2001). El trabajo desarrollado sólo hace referencia a las aves terrestres diurnas debido a las limitaciones impuestas por la metodología utilizada (ver más abajo).

Se ha utilizado una estrategia de muestreo de tipo aleatorio-estratificado, basada en la estima de densidades en diferentes formaciones ambientales, tan homogéneas ecológicamente como fuese posible. El esfuerzo de muestreo se repartió entre estas formaciones ambientales proporcionalmente a su extensión total en la isla. La superficie de cada formación se calculó inicialmente a partir de Anónimo (1980), y fue posteriormente reconsiderada a partir de la cartografía digital ambiental de Canarias (suministrada por la Consejería de Medio Ambiente del Gobierno de Canarias). Las formaciones ambientales consideradas (véase más adelante) son representativas de los gradientes ecológicos existentes en La Palma, incluyendo distintos pisos altitudinales, tipos de paisaje e intensidad de uso del suelo por el hombre. Además, dentro de cada una de las formaciones ambientales distinguidas se muestrearon todas sus localizaciones geográficas en la isla, incluyendo distintas orientaciones cardinales.

\section{MéTodo DE CENSO}

Se ha utilizado el método del transecto lineal para estimar las densidades y preferencias ambientales de las aves estudiadas, así como la variación de la riqueza de especies (véanse los detalles acerca de esta metodología en Bibby et al., 2000; Buckland et al., 2001, 2004). Este es un método que se ha revelado de una gran utilidad en aproximaciones extensivas, siendo muy adecuado en estudios comparados de patrones de distribución, preferencias de hábitat o cambios temporales en los efectivos poblacionales (véanse Gaston y Blackburn, 2000, y referencias en Scott et al., 2002).

Los censos realizados sólo incluyen medios terrestres, habiéndose descartado los acantilados costeros y las zonas húmedas artificiales (balsas, embalses). A continuación se especifica detalladamente el protocolo de muestreo:

- se efectuaron recorridos de $0,5 \mathrm{~km}$ de longitud campo 'a través' o sobre senderos o pistas poco transitadas por el tráfico rodado

- los recorridos se efectuaron a pie a una velocidad 'relativamente' constante de 1-3 (principalmente 2) $\mathrm{km} / \mathrm{h}$

- el punto medio de cada uno de ellos fue georreferenciado mediante GPS (latitud, longitud y altitud)

- se anotaron todos los individuos vistos u oídos a ambos lados del trayecto, estimando la distancia de observación perpendicular al transecto cuando era posible, y distinguiendo si eran contactados a más o menos de $25 \mathrm{~m}$ del observador en relación con la trayectoria del transecto (ver más abajo)

- no se consideraron los pollos volantones observados, de manera que de este modo se intentó evitar sesgos debidos a las diferencias altitudinales y de orientación dentro de la isla asociados con la fenología de la reproducción

- se eligieron las horas del día en las que es máxima la detectabilidad de las aves: 4 primeras horas de la mañana a partir de la salida del sol (7:3011:30 h oficial) y 3 últimas horas de la tarde antes de la puesta (17:30-20:30 h oficial).

En total se han obtenido 437 transectos de 0,5 $\mathrm{km}$. La densidad media de transectos fue de 0,62 transectos por $\mathrm{km}^{2}$ de superficie de la isla. Su localización se muestra en la Figura 1.

Además, se han recorrido $1.400 \mathrm{~km}$ en coche con luz diurna, y se han invertido 45 horas más caminando por diferentes ambientes y partes de la isla. Se han recorrido todas las carreteras asfaltadas de la isla en al menos una ocasión con luz diurna, circulando a una velocidad moderada $(20-50 \mathrm{~km} / \mathrm{h})$. Aunque estos recorridos no fueron efectuados de un modo estandarizado, han sido de utilidad para constatar la abundancia/escasez y la ausencia de las especies de aves terrestres de La Palma.

Con los datos de las distancias de observación de los individuos durante los transectos lineales se pueden obtener las curvas de detectabilidad y las 


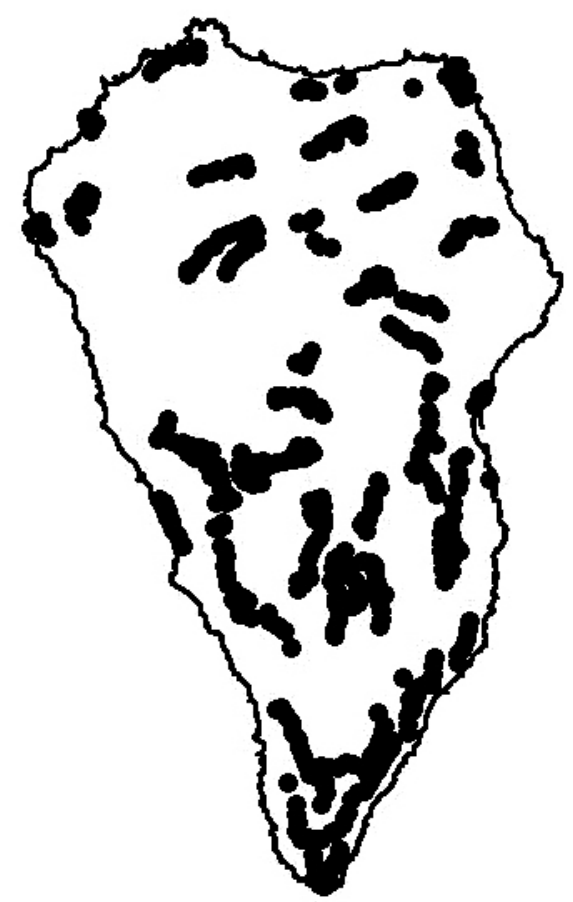

Fig. 1.- Localización de los 437 transectos de $0,5 \mathrm{~km}$ de longitud efectuados en Abril de 2007 en la isla de La Palma. Cada punto señala el centro geográfico de cada transecto.

Fig. 1.- Location of $4370.5 \mathrm{~km}$ transects in La Palma island. Each dot positions the center of the $0.5 \mathrm{~km}$ transects.

distancias eficaces de censo (Buckland et al., 2001, 2004; Thomas et al., 2002). Estos datos son fundamentales para obtener densidades absolutas, y de este modo poder inferir los valores absolutos de individuos existentes en la isla.

Aunque se ha intentado estimar las distancias perpendiculares al trayecto de censo entre las aves y el observador, dicha estima no siempre fue posible debido a la densa cobertura de la vegetación. Por este motivo, se ha utilizado una aproximación más sencilla, que sin embargo produce resultados muy fiables de distancias eficaces de censo (Carrascal, Polo y Seoane en preparación; para más detalles consúltese el Apéndice).

Teniendo en cuenta la distancia eficaz de cada especie (DEC) y la distancia total recorrida al efectuar los 437 realizados en La Palma $(218,5 \mathrm{~km})$, se puede estimar la cantidad de superficie muestreada 'eficazmente' en toda la isla para cada una de las especies consideradas. Esos valores son proporcionados en la Tabla 1.
Tabla 1.- Distancias eficaces de censo (DEC; en m), proporción de individuos observados a menos de $25 \mathrm{~m}$ respecto al recorrido del transecto ( $\mathrm{PROP} \leq 25)$, y superficie censada eficazmente para cada especie en los 437 transectos de $0,5 \mathrm{~km}$ repartidos por toda la isla $\left(\mathrm{CENSOkm}{ }^{2}\right.$; en $\left.\mathrm{km}^{2}\right)$.

Table 1.- Efficient strip width (DEC; in m), proportion of birds detected within belts of $25 \mathrm{~m}$ on both sides of the observer $(\mathrm{PROP} \leq 25)$, and area efficiently surveyed with the 437 transects of $0.5 \mathrm{~km}$ made in La Palma $\left(\mathrm{CENSOkm}^{2}\right.$; in $\mathrm{km}^{2}$ ).

\begin{tabular}{lccc}
\hline spp & PROP $\leq \mathbf{2 5}$ & DEC & CENSO(km $^{2}$ \\
\hline Accipiter nisus & 0.20 & 124.1 & 54.3 \\
Alectoris barbara & 0.39 & 62.4 & 27.3 \\
Anthus berthelotii & 0.42 & 57.6 & 25.2 \\
Burhinus oedicnemus & 0.50 & 47.6 & 20.8 \\
Buteo buteo & 0.11 & 226.8 & 99.1 \\
Acanthis cannabina & 0.59 & 39.2 & 17.1 \\
Columba bolii y junoniae & 0.59 & 39.2 & 17.1 \\
Columba livia & 0.45 & 53.5 & 23.4 \\
Corvus corax & 0.13 & 191.7 & 83.8 \\
Coturnix coturnix & 0.53 & 44.6 & 19.5 \\
Erithacus rubecula & 0.61 & 37.7 & 16.5 \\
Falco tinnunculus & 0.22 & 112.7 & 49.3 \\
Fringilla coelebs & 0.63 & 36.1 & 15.8 \\
Motacilla cinerea & 0.53 & 44.6 & 19.5 \\
Parus caeruleus & 0.55 & 42.7 & 18.7 \\
Passer hispaniolensis & 0.65 & 34.7 & 15.2 \\
Phylloscopus canariensis & 0.47 & 51.0 & 22.3 \\
Pyrrhocorax pyrrhocorax & 0.32 & 76.8 & 33.5 \\
Regulus regulus & 0.89 & 19.0 & 8.3 \\
Scolopax rusticola & 0.63 & 36.1 & 15.8 \\
Serinus canarius & 0.52 & 45.5 & 19.9 \\
Streptopelia decaocto & 0.73 & 29.4 & 12.8 \\
Streptopelia turtur & 0.57 & 40.9 & 17.9 \\
Sylvia atricapilla & 0.42 & 57.6 & 25.2 \\
Sylvia conspicillata & 0.51 & 46.6 & 20.3 \\
Sylvia melanocephala & 0.60 & 38.4 & 16.8 \\
Turdus merula & 0.49 & 48.7 & 21.3 \\
\hline & & &
\end{tabular}

Las densidades absolutas para cada especie se han calculado teniendo en cuenta la siguiente expresión:

$$
\mathrm{D}=\mathrm{N} /(\mathrm{L} \cdot 2 \cdot \mathrm{DEC})
$$

donde $\mathrm{D}$ es la densidad en aves $/ \mathrm{km}^{2}, \mathrm{~N}$ es el número de individuos detectados en la longitud $\mathrm{L}$ de transectos realizados, teniendo en cuenta la distancia eficaz de censo (DEC) de cada especie (dos bandas de esa distancia eficaz a cada lado del observador).

En el caso de Apus unicolor no se pudo calcular la detectabilidad por la imposibilidad práctica de estimar las distancias perpendiculares a la línea del 
transecto debido a su enorme movilidad (para esta especie sólo se proporcionan medidas relativas de abundancia basadas en el número de aves observadas por $\mathrm{km}$ recorrido). Para el caso de Falco peregrinus pelegrinoides se ha utilizado una distancia eficaz aproximada de $175 \mathrm{~m}$, considerando los datos recogidos por los autores principalmente en Fuerteventura y Lanzarote.

En los 437 transectos efectuados en La Palma también se detectó en una sola ocasión a Psittacula krameri, Streptopelia roseogrisea y Carduelis chloris. Estas especies no han sido incluidas en los análisis de datos por ser especies foráneas introducidas por el hombre o de muy reciente colonización (Lorenzo, 2007).

\section{MEdidA DE LA ESTRUCTURA DEL HÁBITAT}

En cada transecto se definieron tres parcelas circulares de $25 \mathrm{~m}$ de radio en las cuales se midieron una serie de variables que describen la estructura del hábitat. Las tres parcelas circulares se localizaron a 125,250 y $375 \mathrm{~m}$ del inicio de cada transecto de $0,5 \mathrm{~km}$. En cada una de ellas se efectuaron estimas de la cobertura (en porcentaje) de roca desnuda, herbáceas en el suelo, estrato arbustivo, estrato arbóreo y de suelo urbanizado, así como de la altura media del matorral, del arbolado y de los edificios. Se establecieron 9 categorías de porcentajes de cobertura $(0,1,2-5,5-12,13-25,25-50,50-75$, 75-95, 95-100\%) para facilitar su estima en el campo. Todas las estimas de cobertura y alturas se efectuaron visualmente, previo entrenamiento entre los distintos participantes en el proyecto para minimizar las diferencias entre ellos. También se caracterizó cada transecto atendiendo a si incluían suelo con uso agropecuario (diversos tipos de cultivo o praderas de siega). La presencia de las principales especies de árboles se codificó atendiendo a si eran o no (si: 1 - no: 0) pinos o especies de laurisilva, o a si los arbustos eran tabaibas (Euphorbia spp), brezos, codesos/escobones, retamas o jaras (varias especies dentro de estas tres últimas categorías que definen biotipos estructurales). Las características de los transectos se obtuvieron mediante el cálculo de la media de las tres estimas efectuadas en ellos.

\section{FORMACIONES AMBIENTALES}

Todos los transectos realizados se han agrupado en once formaciones ambientales considerando su importancia relativa en la isla de La Palma y su relevancia para las aves teniendo en cuenta sus patrones de preferencias de hábitat. Las características de la estructura del hábitat de estas formaciones se muestran en la Tabla 2. En dicha tabla también se indica la superficie aproximada que cada una de estas grandes formaciones ambientales ocupa en la isla de La Palma (obtenida de la cartografía digital ambiental de Canarias, suministrada por la Consejería de Medio Ambiente del Gobierno de Canarias). Las once formaciones ambientales son las siguientes: lavas recientes, tabaibales, brezal-fayal, laurisilva, pinar-monteverde, pinares, matorrales alta montaña, medianías con matorral en regeneración (uso tradicional agropecuario abandonado en gran medida), medianías con uso tradicional del suelo y casas dispersas, cultivos intensivos (plataneras y cultivos de tomate y proteas), areas urbanas más o menos dispersas.

\section{ANÁLISIS DE DATOS}

La amplitud de distribución geográfica de las aves en toda la isla se estimó como la frecuencia de aparición de cada especie en los 437 transectos de $0,5 \mathrm{~km}$ efectuados.

Para estimar la densidad media de cada especie en la isla, y su intervalo de confianza al $90 \%$, se utilizó un procedimiento de remuestreo de los 437 transectos efectuados. Dicho remuestreo se hizo proporcionalmente a la superficie que cada formación ambiental ocupa en la isla de La Palma (véase Carrascal et al., 2006, 2007 para una aproximación similar). Para ello se efectuó una extracción al azar de transectos dentro de cada formación ambiental proporcional a la superficie de cada una de ellas en La Palma, de manera que se seleccionase, como máximo, las $2 / 3$ partes de los transectos realizados en cada formación. Esta restricción redujo la muestra de transectos seleccionados al azar a 130 del conjunto de los 437 transectos efectuados en La Palma (i.e., 29,7\% del total de transectos disponibles). Tras efectuar cada extracción al azar de los 130 transectos, se calculó la densidad media insular de cada especie dividiendo el número de aves observadas en ellos por la superficie que se hubiese censado considerando la distancia eficaz de censo (DEC; 130/2 × 2 x [DEC/1000] $\mathrm{km}^{2}$ ). Este procedimiento se repitió 1000 veces, para después extraer los percentiles $5 \%$ y $95 \%$ de la distribución de los 1000 valores de densidad obtenidos para cada especie, y generar el correspondiente intervalo de confianza al $90 \%$ en toda la isla. Este proceso se desarrolló con MS-Excel utilizando macros para la selección de muestras y los cálculos subsiguientes.

Teniendo en cuenta los transectos efectuados en cada formación vegetal, y la distancia eficaz de censo de cada especie, se estimó la densidad en los once medios distinguidos. 


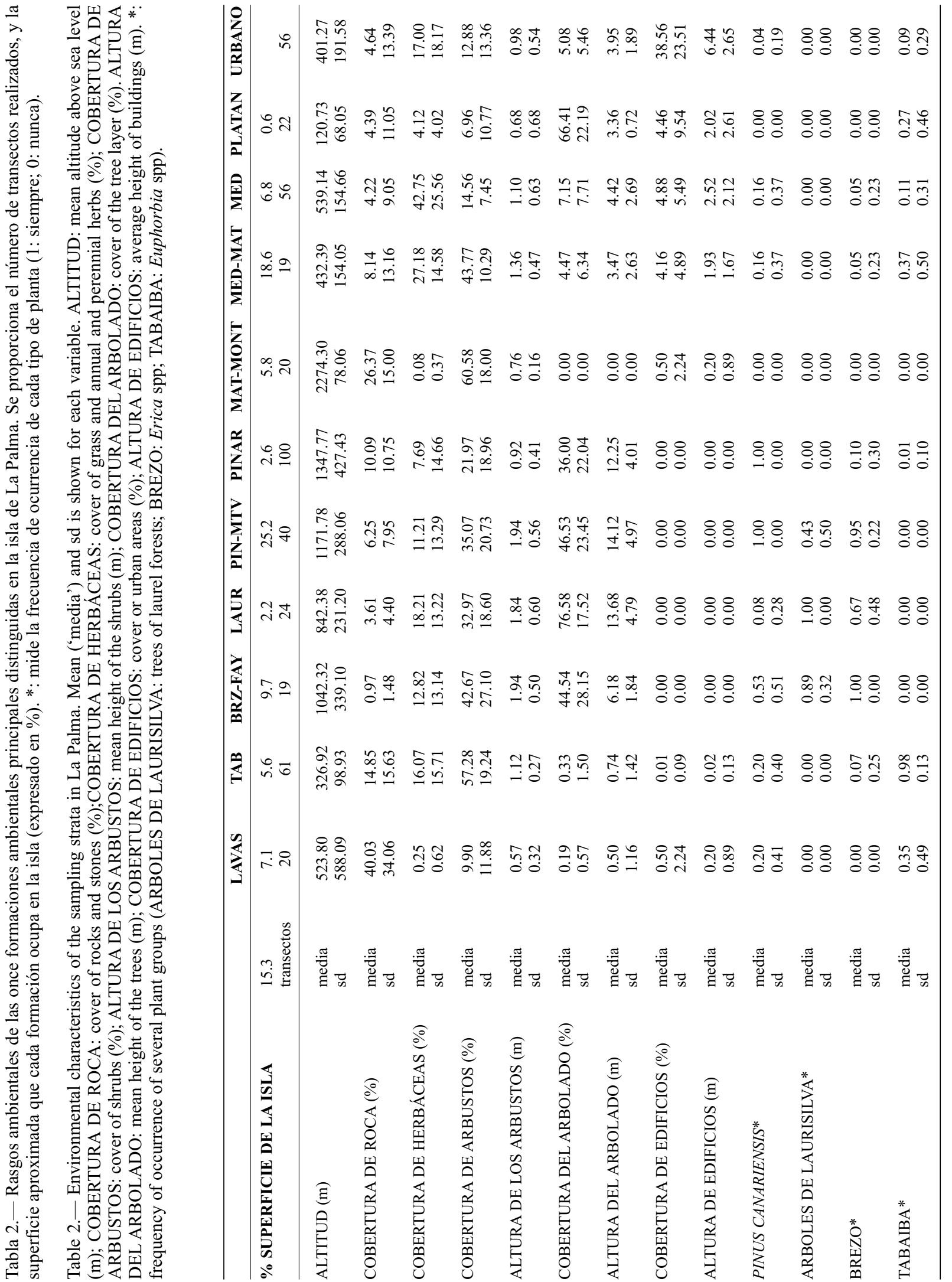


Dichos valores de densidad media, y mínimos - máximos de confianza al 90\%, se multiplicaron por la superficie de La Palma para de este modo obtener la estima de individuos existentes en la isla.

La amplitud de hábitat ( $\mathrm{AH}$ ) de las especies en las once formaciones ambientales principales se calculó mediante la siguiente fórmula:

$$
\mathrm{AH}=\left(1 / \sum \mathrm{p}_{\mathrm{i}}^{2}\right) / 11
$$

donde $\mathrm{p}_{\mathrm{i}}$ es la proporción de la densidad en la formación $i$ considerando la suma de densidades en todos los 11 ambientes. Este índice varía entre $0 \mathrm{y}$ 1 , de manera que a mayor valor del índice le corresponde una mayor amplitud de hábitat de la especie (i.e., mayor valencia ecológica). Esta misma estima de amplitud también se calculó considerando solamente los siete ambientes naturales sin uso humano (AHnat; excluyendo las áreas urbanas, los cultivos intensivos y los dos tipos de medianías).

La riqueza de especies es un fenómeno dependiente de la inversión en esfuerzo de muestreo, de manera que el número de especies diferentes registradas va creciendo asintóticamente según aumenta la superficie censada. La asíntota final es el número de especies total existente en la región de estudio. Para medir el patrón acumulado de especies según aumentaba la superficie muestreada se siguió un proceso de remuestreo utilizando todos los datos disponibles. Para ello se extrajo al azar 250 transectos de los 437 realizados en toda la isla, de manera que dicha extracción fuese proporcional a la superfcie que cada formación ambiental ocupaba en La Palma. Una vez que se habían obtenido estas 250 muestras, se extrajo una al azar y se contó el número de especies observadas. Luego se extrajeron dos transectos al azar dentro de la muestra de 250 transectos seleccionados en el proceso de muestreo, anotándose el número de especies presentes en esos dos transectos. Este proceso se repitió con grupos crecientes de transectos ( $\sin$ reemplazo) de tres, cuatro, cinco, seis, ...50, ...100, ... y así hasta los 250 transectos elegidos al azar. Este proceso de remuestreo, y de aglomeración de transectos al azar, se repitió 100 veces. De este modo, se pudo obtener el patrón acumulativo de especies conforme se iba incrementando el esfuerzo de muestreo (y por tanto la superficie censada en La Palma), y su intervalo de confianza al $95 \%$. Se utilizaron macros escritas en MS-Excel para la selección de muestras y los cálculos subsiguientes.
Este mismo proceso de aglomeración de muestras al azar se efectuó también con los transectos realizados dentro de cada una de las once formaciones ambientales, repitiéndose en cada una de ellas 100 veces.

El número medio especies (resultante de las 100 aglomeraciones distintas) encontradas a medida que se incrementa el número de transectos se modelizó utilizando regresión con el programa share-ware CurveExpert 1.3 disponible en internet (http://curveexpert.webhop.net/). El programa CurveExpert muestra, de entre un enorme abanico de modelos matemáticos posibles, todos aquéllos en los que la curva se ajusta razonablemente bien a los datos de partida, y además los presenta ordenados de mayor a menor proporción de varianza explicada. El modelo Morgan-Mercer-Flodin MMF fue siempre el más adecuado de todos los ofrecidos. Como medida comparada de la riqueza de especies en cada formación ambiental se utilizó el número de especies que los modelos de regresión MMF predecían al efectuar 20 transectos de $0,5 \mathrm{~km}$ de longitud (i.e., $10 \mathrm{~km}$ de censo). También se calcularon los valores que la derivada de las curvas MMF tenían a los 20 transectos realizados. Este valor mide la pendiente de la curva con ese esfuerzo de muestreo estándar y comparable, y supone una medida de las expectativas de encontrar nuevas especies.

Los factores influyentes sobre la riqueza de especies a escala local (coberturas y alturas de los distintos estratos vegetales y de las edificaciones) se exploraron mediante regresiones de mínimos cuadrados parciales (regresiones PLS - de 'Partial Least Squares regressions'; Frank y Friedman, 1993; Tobias, 2003; Abdi, 2007), una herramienta analítica especialmente útil para evaluar el efecto de variables predictoras altamente correlacionadas entre sí. La regresión PLS es una extensión del análisis de regresión múltiple lineal en donde se obtienen factores multivariantes ortogonales entre sí que son combinaciones lineales de los predictores originales (semejantes a los obtenidos en un análisis de las componentes principales), pero condicionados a maximizar la varianza explicada en la variable respuesta. Puesto que localidades adyacentes pueden albergar riquezas de especies muy semejantes, más por su mera proximidad geográfica que por su estructura de hábitat, el efecto de este contagio espacial fue controlado incluyendo en las regresiones PLS términos polinomiales de la latitud y longitud de cada transecto (Legendre, 1993).

Los análisis estadísticos fueron efectuados con Statistica 6.0 (StatSoft 2001). 


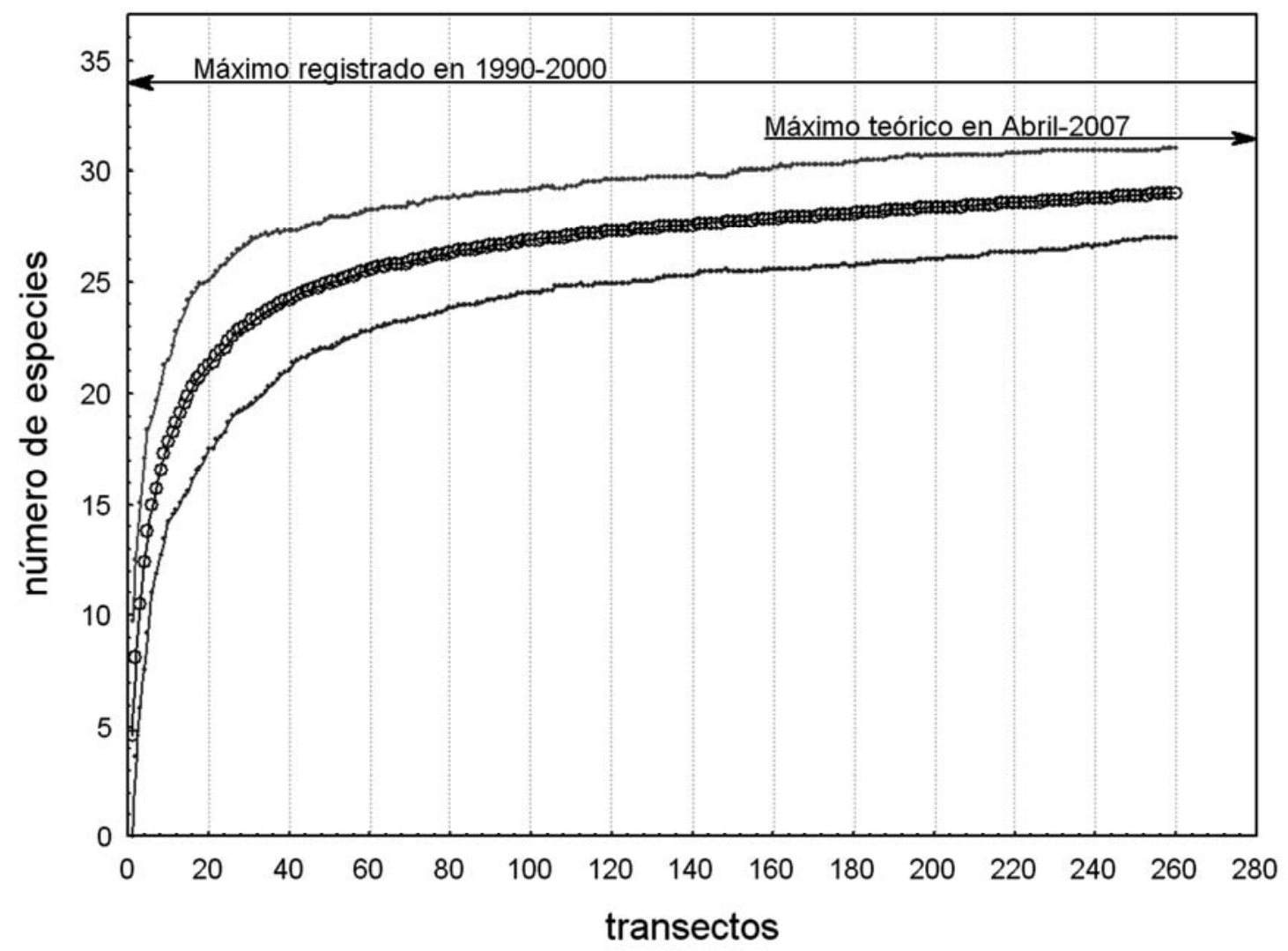

Fig. 2.- Variación del número de especies de aves terrestres diurnas reproductoras en la isla de La Palma en función del número de transectos realizados. El patrón descrito se ha obtenido por un proceso de remuestreo-aglomeración aleatorio, extrayendo los transectos proporcionalmente a la disponibilidad en la isla de las once formaciones ambientales consideradas (100 procesos de aleatorización efectuados). Para más detalles véase el texto. Se proporciona el número medio de especies, el intervalo de confianza al 95\%, y distintos máximos registrados de riqueza de especies en La Palma.

Fig. 2.- Cumulative curve of species of terrestrial birds detected with increasing sampling effort (number of $0.5 \mathrm{~km}$ transects) in La Palma island. The curve and 95\% confidence intervals were obtained by means of 100 permutation and bootstrapping processes. The arrows show the maximum number of breeding bird species recorded in the last 25 years (Martín \& Lorenzo, 2001), and the theoretical maximum provided by the model in the whole area of La Palma island.

\section{Resultados y Discusión}

\section{RiQUEZA DE ESPECIES A ESCALA INSULAR}

Considerando las distancias eficaces de censo (ver Tabla 1), los 437 transectos de $500 \mathrm{~m}$ de longitud efectuados $(218,5 \mathrm{~km}$ recorridos) cubren entre el $1,2 \%$ (caso de Regulus regulus, $8,3 \mathrm{~km}^{2}$ ) y el $14,0 \%$ (caso de Buteo buteo, $99,1 \mathrm{~km}^{2}$ ) de la superficie de toda la isla. Teniendo en cuenta a todas las especies, el esfuerzo de muestreo realizado ha cubierto eficazmente un promedio del $4,0 \%$ de la superficie de La Palma.

La Figura 2 ilustra el patrón de variación de la riqueza de especies de aves terrestres en la isla de
La Palma en función del esfuerzo de muestreo. Existe una relación muy intensa entre el número de transectos realizados y el número de especies observadas $\left(\mathrm{R}^{2}=0,9997, \mathrm{p}<<0,001\right)$ utilizando el modelo de regresión MMF:

$$
\begin{aligned}
\text { Especies }= & \left(-10,2 \cdot 1,89+31,7 \cdot \text { transectos }^{0,582}\right) / \\
& \left(1,89+\text { transectos }^{0,582}\right)
\end{aligned}
$$

La Figura 2 claramente indica que la variación de la riqueza de especies con la superficie de la isla se estabiliza bastante rápidamente, de manera que una gran parte de las especies de aves reproductoras suelen distribuirse ampliamente por toda la isla, y son muy pocas las especies muy raras que 
Tabla 3.- Modelos de regresión MMF de la relación [número de especies - transectos realizados] en las once formaciones ambientales distinguidas en la isla de La Palma. Los modelos se han obtenido de datos generados mediante un proceso de remuestreo-aglomeración aleatorio del total de transectos en cada formación ambiental (100 procesos de aleatorización efectuados). $a, b$, $c$ y $d$ son los cuatro coeficientes de regresión del modelo MMF. $\mathrm{R}^{2}$ : porcentaje de la varianza explicado por el modelo. SPP20: número de especies estandarizado a 20 transectos. pte20: pendiente de la curva a 20 transectos efectuados (a mayor pendiente, mayor espectativa de encontrar nuevas especies en sucesivos transectos). Para más detalles véase el Material y Métodos. El modelo de regresión MMF tiene la siguiente expresión ( $y$ : número de especies; $x$ : número de transectos): $\mathrm{y}=\left(a \cdot b+c \cdot \mathrm{x}^{d}\right) /\left(b+\mathrm{x}^{d}\right)$.

Table 3.- Morgan-Mercer-Flodin regression models relating the cumulative number of bird species detected with the number of $0.5 \mathrm{~km}$ transects censused in eleven environmental units of La Palma island. $a, b, c$ and $d$ are the regression parameters of the equation $\mathrm{y}=\left(a \cdot b+c \cdot \mathrm{x}^{d}\right) /\left(b+\mathrm{x}^{d}\right)$, where $\mathrm{y}$ is the number of bird species, and $\mathrm{x}$ the number of $0.5 \mathrm{~km}$ transects. $\mathrm{R}^{2}$ : proportion of the variance accounted for by each regression model. SPP20: number of bird species predicted in 20 transects of $0.5 \mathrm{~km}$. pte 20 : slope of the regression model at 20 transects of $0.5 \mathrm{~km}$.

\begin{tabular}{lccccccc}
\hline & $\boldsymbol{a}$ & $\boldsymbol{b}$ & $\boldsymbol{c}$ & $\boldsymbol{d}$ & $\mathbf{R}^{\mathbf{2}}$ & SPP20 & pte20 \\
\hline LAVAS RECIENTES & & & & & & & \\
TABAIBALES & 0.2 & 42.2 & 95.1 & 0.63 & 0.993 & 12.90 & 0.35 \\
BREZAL-FAYAL & -29.5 & 2.6 & 97.2 & 0.15 & 0.999 & 17.85 & 0.22 \\
LAURISILVA & -32.6 & 1.2 & 52.0 & 0.17 & 0.999 & 17.24 & 0.18 \\
PINAR-MONTEVERDE & 2.2 & 2.5 & 18.2 & 0.52 & 0.998 & 12.66 & 0.09 \\
PINARES & -3.9 & 1.9 & 25.6 & 0.48 & 0.999 & 16.17 & 0.15 \\
MATORRALES ALTA MONTAÑ & 0.2 & 3.7 & 22.2 & 0.76 & 0.999 & 16.15 & 0.17 \\
MEDIANIAS CON MATORRAL & 2.4 & 12.0 & 9.9 & 1.47 & 0.999 & 8.94 & 0.06 \\
MEDIANÍAS & -2.6 & 4.5 & 44.9 & 0.48 & 1.000 & 20.32 & 0.28 \\
PLATANERAS & 2.2 & 6.5 & 32.2 & 0.80 & 1.000 & 21.04 & 0.28 \\
AREAS URBANAS & -7.8 & 2.1 & 32.2 & 0.26 & 0.998 & 12.78 & 0.13 \\
\hline
\end{tabular}

contribuyen de modo destacado a la riqueza global de la isla.

Martín y Lorenzo (2001) proporcionan un listado de 34 especies autóctonas de aves terrestres diurnas reproductoras en La Palma. Con los 437 transectos realizados $(218,5 \mathrm{~km}$ recorridos), que cubrirían solamente el 4,0\% de la superficie de la isla, se han observado 30 especies de aves terrestres reproductoras. Las cuatro especies no observadas, ni en los transectos estandarizados, ni en otras prospecciones por toda la isla $(1400 \mathrm{~km}$ recorridos en coche con luz diurna y 45 horas de recorridos a pié por diferentes ambientes) han sido Upupa epops, Carduelis carduelis, Petronia petronia y Miliaria calandra. Martín y Lorenzo (2001) indican que estas especies se han convertido en muy raras desde la década de 1980, existiendo a finales del siglo XX escasísimas localidades donde podían ser observadas.

Asumiendo una superficie media prospectada en cada transecto de $0,065 \mathrm{~km}^{2}$ (teniendo en cuenta el promedio de las distancias eficaces de censo para las 30 especies observadas), el modelo de variación de la riqueza de especies en función del esfuerzo de muestreo predice 31,3 especies de aves terrestres para los $708 \mathrm{~km}^{2}$ de la isla de La Palma. Esto es, el
$96 \%$ de las teóricamente existentes. Por tanto, es muy probable que de las cuatro especies indicadas por Martín y Lorenzo (2001) que no se han observado en este estudio, tres hayan ya desaparecido como reproductores estables y una aun esté presente con escasísimos efectivos.

\section{VARIACIÓN DE LA RIQUEZA DE ESPECIES DENTRO DE} LA ISLA.

En la Tabla 3 se exponen los resultados de los modelos de regresión MMF que relacionan la riqueza de especies con el número de transectos realizados. Todos los modelos son altamente significativos $(\mathrm{p}<<0,001)$ y explican más del $99 \%$ de la variación observada en la relación especies-esfuerzo de muestreo.

A igualdad de número de transectos muestreados (medida estándar de riqueza a 20 transectos efectuados), el ambiente con menor biodiversidad aviar es, con diferencia, el matorral altimontano (8,9 especies). Es más, la pendiente de la curva de rarefacción al muestrear 20 transectos es la más pequeña $(0,06)$, lo cual indica que las expectativas de encontrar nuevas especies al muestrear más superficie son muy pequeñas (i.e., la riqueza de 
especies está muy estabilizada). Con mayores valores de riqueza de especies de aves reproductoras terrestres, aunque relativamente bajos dentro del contexto de La Palma (de 12 a 13 especies en 20 transectos, o $1,3 \mathrm{~km}^{2}$ de superficie) están formaciones tan dispares como las plataneras, la laurisilva y las formaciones volcánicas recientes. De estos tres ambientes, la laurisilva madura es aquella formación con menos espectativas de encontrar nuevas especies al muestrear más superficie (pendiente de la curva al muestrear 20 transectos $=0,09$; i.e., casi todas las especies que pueblan las laurisilvas de La Palma están presentes en una muestra tan reducida como 20 transectos o una superficie de $1,3 \mathrm{~km}^{2}$ ). Por el contrario, en las formaciones volcánicas recientes las espectativas de añadir nuevas especies al listado de las que habitan en ellas son muy altas, siendo la pendiente de la curva, a 20 transectos efectuados, la mayor de las medidas dentro de la isla $(0,35)$. Este hecho viene motivado por el carácter ecotónico de esta formación ambiental, que está presente desde el nivel del mar hasta casi los 2000 $\mathrm{m}$ de altitud, y que atraviesa en forma de coladas una gran variedad de ambientes (e.g., pinares, tabaibales, medianías).

Un tercer grupo de formaciones vegetales atendiendo a los valores medios de riqueza de especies incluye formaciones también tan dispares como los tabaibales, los fayales-brezales, los pinares, las transiciones pinar-monteverde y las áreas urbanas (de 16 a 18 especies en 20 transectos,o una superficie de $1,3 \mathrm{~km}^{2}$ ). Todos ellos tienen espectativas medio-altas de añadir nuevas especies según se prosigue añadiendo superficie muestreada (rango de los valores de pendiente de las curvas de rarefacción a un número estándar de 20 transectos: 0,15-0,22), aunque en los que más aumenta la riqueza de aves al inventariar más superficie son los tababibales y las áreas urbanas.

Por último, las formaciones ambientales con mayor riqueza de especies, que al mismo tiempo mantienen altas expectativas de incluir nuevas especies según se aumenta la superficie muestreada, son las medianías dedicadas a la agricultura y con casas dispersas, independientemente del desarrollo del matorral en regeneración (20-21 especies por 1,3 $\mathrm{km}^{2}$ o 20 transectos muestreados, y pendientes de la curva, a 20 transectos censados, de 0,28).

La diversidad de aves, estimada mediante el índice de Shanon (Tabla 4), muestra una variación entre los once ambientes considerados que es consistente con la variación observada en la riqueza de especies estandarizada $(r=0,872, p=0,0005$; utilizando el logaritmo neperiano de SPP20).
Tabla 4.- Densidad total (aves $/ \mathrm{km}^{2}$ ) y diversidad (en nats; usando logaritmos neperianos en la fórmula de Shanon) de las comunidades de aves de las once formaciones ambientales principales de la isla de La Palma.

Table 4.- Total density (birds $/ \mathrm{km}^{2}$ ) and diversity (Shanon index) of the bird communities inhabiting eleven environmental units of La Palma island.

\begin{tabular}{lcc}
\hline & ${\text { AVES } / \mathbf{~ K M}^{2}}^{2}$ & DIVERSIDAD \\
\hline LAVAS RECIENTES & 57.6 & 1.84 \\
TABAIBALES & 287.1 & 2.14 \\
BREZAL-FAYAL & 630.2 & 1.90 \\
LAURISILVA & 656.9 & 1.94 \\
PINAR-MONTEVERDE & 509.0 & 2.01 \\
PINARES & 314.4 & 1.97 \\
MATORRALES ALTA MONTAÑA & 152.7 & 1.43 \\
MEDIANÍAS CON MATORRAL & 468.4 & 2.05 \\
MEDIANÍAS & 475.1 & 2.09 \\
PLATANERAS & 232.2 & 1.91 \\
AREAS URBANAS & 459.5 & 2.08 \\
\hline
\end{tabular}

Sin embargo, la densidad total de aves manifiesta un patrón de variación ambiental muy distinto, ya que dicho parámetro no está significativamente relacionado ni con la diversidad $(\mathrm{r}=0,447, \mathrm{p}=$ $0,168)$ ni con la riqueza estandarizada (SPP20; $r=$ $0,471, p=0,144)$. La densidad total de aves (Tabla 4) alcanza sus máximos valores en el monteverde de laurisilva $\left(657\right.$ aves $\left./ \mathrm{km}^{2}\right)$ y fayal-brezal $(630$ aves $/ \mathrm{km}^{2}$ ). La transición pinar-laurisilva también mantiene una elevada densidad de aves (509 aves $/ \mathrm{km}^{2}$ ). De los restantes medios naturales, los matorrales de alta montaña $\left(153\right.$ aves $\left./ \mathrm{km}^{2}\right)$ y las lavas recientes $\left(58\right.$ aves $/ \mathrm{km}^{2}$ ) mantienen las avifaunas menos densas. Los medios antropizados muestran valores medio-altos de densidad aviar, destacando las plataneras por la menor abundancia de aves.

La Tabla 5 muestran los resultados del análisis de regresión por el método de los cuadrados mínimos parciales aplicado a la variación del número de especies en los transectos de $500 \mathrm{~m}$ (i.e., riqueza local). Se obtienen dos componentes significativos que en conjunto explican el $36,5 \%$ de la variación observada. La primera componente (COMP-1 en Tabla 5; 33,0\% de la varianza explicado, $\mathrm{r}=0,575$, $\mathrm{p}<0,001$ ), se asocia con la altitud sobre el nivel del mar y con la latitud tanto de modo lineal como cuadrático, definiendo un patrón de covariación con la riqueza no lineal. La riqueza local de especies aumenta desde el nivel del mar hasta alcanzar un 
Tabla 5.- Resultado del análisis de regresión por el método de mínimos cuadrados ('partial least squares regression analysis') con la variación del número de especies de aves terrestres diurnas en transectos de $0,5 \mathrm{~km}$ de longitud. Los coeficientes de cada variable indican sus pesos en cada factor. Se marcan en negrita aquellas variables asociadas significativamente con cada factor y que además retienen más de un $5 \%$ de su contenido informativo.

Table 5.- Results of the partial least squares regression analysis (PLSR) carried out with the local species richness (number of bird species detected in $0.5 \mathrm{~km}$ transects). The two components shown (COMP-1 and COMP-2) define two gradients of environmental variation that are significantly related to local species richness $(p<0.001)$. LONGITUD and LATITUD are the longitude and latitude of $0.5 \mathrm{~km}$ transects. For the other variables see Table 2. Sample size is 437 transects of $0.5 \mathrm{~km}$ made in La Palma. PLSR weights significant and whose squares are larger than 0.05 are shown in bold type.

\begin{tabular}{lcc}
\hline & COMP-1 & COMP-2 \\
\hline & 0.073 & $\mathbf{- 0 . 2 6 7}$ \\
LONGITUD & $\mathbf{0 . 3 0 9}$ & 0.080 \\
LATITUD & 0.077 & $\mathbf{- 0 . 2 6 8}$ \\
LONGITUD $^{2}$ & $\mathbf{0 . 3 0 9}$ & 0.079 \\
LATITUD $^{2}$ & 0.122 & $\mathbf{- 0 . 2 6 0}$ \\
LONGITUD X LATITUD $^{\text {ALTITUD }}$ & $\mathbf{- 0 . 2 6 1}$ & 0.04 \\
ALTITUD $^{2}$ & $\mathbf{- 0 . 3 3 0}$ & -0.107 \\
COBERTURA DE ROCA & $\mathbf{- 0 . 3 9 2}$ & -0.089 \\
COBERTURA DE HERBÁCEAS & $\mathbf{0 . 4 1 2}$ & 0.211 \\
COBERTURA DE ARBUSTOS & 0.157 & $\mathbf{0 . 5 8 9}$ \\
ALTURA DE LOS ARBUSTOS & $\mathbf{0 . 3 7 8}$ & $\mathbf{0 . 2 8 1}$ \\
COBERTURA DEL ARBOLADO & 0.084 & 0.016 \\
ALTURA DEL ARBOLADO & 0.135 & $\mathbf{0 . 3 0 4}$ \\
COBERTURA DE SUELO AGRÍCOLA & 0.155 & $\mathbf{- 0 . 4 1 7}$ \\
COBERTURA DE EDIFICIOS & 0.160 & -0.045 \\
ALTURA DE EDIFICIOS & 0.203 & -0.125 \\
\hline
\end{tabular}

máximo a altitudes medias, para luego bajar hasta los valores menores a elevadas altitudes; en relación con la latitud, aumenta desde el sur de la isla al norte, aunque pasa por un mínimo a latitudes medias dentro de la isla. Ambas variables, latitud y altitud, definen mínimos de riqueza en las zonas centrales de la isla de mayor altitud, un aumento desde el sur al norte de la isla, y máximos a altitudes medias (principalmente de 600 a $1.000 \mathrm{~m}$ s.n.m.). El número de especies por $0,5 \mathrm{~km}$ de transecto disminuyó al aumentar la cobertura de roca desnuda sobre el suelo, y aumentó con la cobertura de herbáceas y la altura de los arbustos. La segunda componente (COMP-2) tan solo explicó el 3,5\% de la variación observada en la riqueza local de especies $(r=0,227, p<0,001)$. Incluye un efecto geográfico relacionado con la longitud (riqueza de especies que disminuye del noroeste de la isla al sureste), y asociado a un aumento del desarrollo del estrato arbustivo (en cobertura y altura) y arbóreo (altura del arbolado), y una menor intensidad de uso agrícola del suelo. Hay que destacar que las dos variables que definen la intensidad de urbanización no se asociaron con la riqueza de especies.

En resumen, la variación local de la riqueza de especies en la isla de La Palma tiene una importante componente geográfica (aumento del norte al sur y del este al oeste, con valores máximos a altitudes medias), asociándose además con el desarrollo de la vegetación especialmente en los estratos arbustivos y herbáceo, y estando negativamente influida por el desarrollo de la agricultura pero no por el urbanismo.

AMPLITUD DE DISTRIBUCIÓN, DENSIDAD Y TAMAÑO DE POBLACIÓN A ESCALA INSULAR

La Tabla 6 muestra los resultados medios de frecuencia de aparición, abundancia relativa y densidad media de las especies de aves para toda la isla de La Palma. La especie más ampliamente distribuida fue Phylloscopus canariensis que estuvo presente en el $84,3 \%$ de los transectos efectuados. Otras especies también ampliamente distribuidas que aparecieron en al menos una tercera parte de los transectos fueron Turdus merula, Serinus canaria y Sylvia atricapilla. Otras quince especie pueden considerarse comunes al haber aparecido en, al menos, el 5\% de los transectos (por orden decreciente de frecuencia de aparición: Regulus regulus ellenthalerae, Columba livia [domestica], Fringilla coelebs, Parus caeruleus, Falco tinnunculus, Erithacus rubecula, Apus unicolor, Anthus berthelotii, Sylvia melanocephala, Pyrrhocorax pyrrhocorax, Columba junoniae, Columba bolii, Streptopelia turtur, Alectoris barbara y Motacilla cinerea). Ocho especies son raras al estar presentes sólo en el 5-0,5\% de los transectos de 0,5 km efectuados (por orden decreciente de frecuencia de aparición: Streptopelia decaocto, Sylvia conspicillata, Carduelis cannabina, Buteo buteo, Corvus corax, Accipiter nisus, Scolopax rusticola y Coturnix coturnix). Por último, Falco [peregrinus] pelegrinoides, Burhinus oedicnemus y Passer hispaniolensis pueden considerarse muy raras al estar muy escasamente repartidas por la isla.

Las especies que alcanzan mayores densidades medias en La Palma, de más de 25 aves $/ \mathrm{km}^{2}$, son Phylloscopus canariensis, Regulus regulus ellenthalerae, Turdus merula, Serinus canaria y Columba livia. Por el contrario, las especies con menores den- 
Tabla 6.- Frecuencias de aparición de las especies en transectos de 0,5 km, y densidades absolutas medias en la isla de La Palma (junto con sus intervalos de confianza al $90 \%$ obtenidos por remuestreo).

Table 6.- Frequency of occurrence in $0.5 \mathrm{~km}$ transects and average population density of terrestrial bird species in La Palma island. IC 90\%: bootstrapped $90 \%$ confidence intervals.

\begin{tabular}{|c|c|c|c|c|c|c|}
\hline & \multicolumn{3}{|c|}{ FRECUENCIA } & \multicolumn{3}{|c|}{ AVES / KM ${ }^{2}$} \\
\hline & media & IC $90 \%$ & IC $90 \%$ & media & IC $90 \%$ & IC $90 \%$ \\
\hline Accipiter nisus & 0.019 & 0.008 & 0.038 & 0.16 & 0.06 & 0.31 \\
\hline Alectoris barbara & 0.057 & 0.031 & 0.085 & 1.30 & 0.61 & 1.96 \\
\hline Anthus berthelotii & 0.176 & 0.146 & 0.200 & 5.72 & 4.31 & 7.28 \\
\hline Apus unicolor & 0.178 & 0.138 & 0.215 & & & \\
\hline Burhinus oedicnemus & 0.004 & 0.000 & 0.015 & 0.09 & 0.00 & 0.32 \\
\hline Buteo buteo & 0.037 & 0.015 & 0.054 & 0.18 & 0.07 & 0.27 \\
\hline Carduelis cannabina & 0.038 & 0.019 & 0.062 & 4.24 & 0.78 & 8.34 \\
\hline Columba bolii & 0.095 & 0.077 & 0.115 & 6.12 & 4.70 & 7.63 \\
\hline Columba junoniae & 0.115 & 0.092 & 0.138 & 6.77 & 4.89 & 8.81 \\
\hline Columba livia & 0.273 & 0.231 & 0.315 & 28.15 & 19.55 & 37.24 \\
\hline Corvus corax & 0.033 & 0.015 & 0.054 & 0.21 & 0.08 & 0.35 \\
\hline Coturnix coturnix & 0.005 & 0.000 & 0.015 & 0.11 & 0.00 & 0.35 \\
\hline Erithacus rubecula & 0.187 & 0.162 & 0.223 & 13.21 & 10.34 & 16.02 \\
\hline Falco pelegrinoides & 0.001 & 0.000 & 0.008 & 0.01 & 0.00 & 0.06 \\
\hline Falco tinnunculus & 0.196 & 0.154 & 0.238 & 1.96 & 1.57 & 2.40 \\
\hline Fringilla coelebs & 0.263 & 0.223 & 0.300 & 18.84 & 15.10 & 22.75 \\
\hline Motacilla cinerea & 0.049 & 0.031 & 0.069 & 1.56 & 0.69 & 2.43 \\
\hline Parus caeruleus & 0.255 & 0.215 & 0.292 & 10.95 & 8.76 & 13.59 \\
\hline Passer hispaniolensis & 0.004 & 0.000 & 0.015 & 0.23 & 0.00 & 1.10 \\
\hline Phylloscopus canariensis & 0.843 & 0.815 & 0.869 & 92.59 & 86.09 & 98.98 \\
\hline Pyrrhocorax pyrrhocorax & 0.129 & 0.092 & 0.162 & 7.56 & 3.45 & 12.87 \\
\hline Regulus regulus & 0.308 & 0.277 & 0.338 & 45.27 & 37.36 & 53.83 \\
\hline Scolopax rusticola & 0.009 & 0.000 & 0.015 & 0.23 & 0.00 & 0.42 \\
\hline Serinus canarius & 0.440 & 0.392 & 0.492 & 42.21 & 34.99 & 49.70 \\
\hline Streptopelia decaocto & 0.040 & 0.023 & 0.062 & 2.76 & 1.04 & 4.96 \\
\hline Streptopelia turtur & 0.081 & 0.054 & 0.115 & 2.51 & 1.49 & 3.55 \\
\hline Sylvia atricapilla & 0.355 & 0.315 & 0.392 & 17.18 & 14.61 & 19.88 \\
\hline Sylvia conspicillata & 0.039 & 0.015 & 0.062 & 1.23 & 0.50 & 2.00 \\
\hline Sylvia melanocephala & 0.162 & 0.131 & 0.192 & 7.82 & 5.80 & 10.00 \\
\hline Turdus merula & 0.482 & 0.438 & 0.523 & 40.65 & 36.07 & 45.54 \\
\hline
\end{tabular}

sidades medias en toda la isla de La Palma, con menos de 0,25 aves $/ \mathrm{km}^{2}$, son Scolopax rusticola, Passer hispaniolensis, Corvus corax, Buteo buteo, Accipiter nisus, Coturnix coturnix, Burhinus oedicnemus y Falco [peregrinus] pelegrinoides.

Teniendo en cuenta las densidades ecológicas medias en toda la isla, y la superficie de la misma, en la Tabla 7 se proporciona el tamaño de población de cada especie y su intervalo de confianza al 90\%. No ha sido posible efectuar esta estima para Apus unicolor, debido a la imposibilidad de calcular su detectabilidad. Por otro lado, para las especies más raras sólo se puede asumir con certeza que sus efectivos son muy pequeños, debido al escaso tamaño muestral. Se indica cuán fiables son las estimas numéricas calculadas en función de la variabilidad de su intervalo de confianza al 90\%. También se proporciona la categoría de tamaño de población que con una elevada probabilidad tiene cada especie en la isla de La Palma. Esta escala pretende proporcionar estimas creíbles de cuáles deben ser los efectivos poblacionales más probables de cada especie en la isla de La Palma.

En la Tabla 8 se proporcionan los valores de densidad de las 30 especies de aves terrestres reproductoras diurnas en La Palma (para Apus unicolor sólo se proporcionan índices relativos de abundancia). La lectura vertical de dicha tabla muestra a las especies que son más abundantes en cada medio, y por tanto que los caracterizan. Por otro lado, su lectura trans- 
Tabla 7.- Estimas del tamaño de población (individuos) de las especies de aves terrestres diurnas en la isla de La Palma. Media, Máx-90\%: estima media e intervalo de confianza superior al 90\%. MF: estimas muy fiables y con poca variabilidad; F: fiables pero con marcada variabilidad ofrecida por su intervalo de confianza al 90\%; PF: poco fiables utilizando criterios estadísticos muy exigentes relativos al número de contactos para estimar la detectabilidad, número de aves observadas y amplitud del intervalo de confianza al $90 \%$.

Table 7.- Global population estimates (individuals) of terrestrial bird species in La Palma island. Media, Máx-90\%: average and upper limit of the $90 \%$ confidence interval. MF: very reliable estimate; F: reliable according to the low variability shown by the $90 \%$ confidence interval; PF: low reliability according to the large variability shown by the $90 \%$ confidence interval. Escala máx. Fiabilidad: probable population

\begin{tabular}{|c|c|c|c|c|}
\hline & Media & Máx-90\% & fiabilidad & Escala máx. fiabilidad \\
\hline Phylloscopus canariensis & 65554 & 70079 & MF & $50.000-100.000$ \\
\hline Regulus regulus & 32051 & 38109 & MF & $25.000-50.000$ \\
\hline Serinus canarius & 29883 & 35188 & MF & $25.000-50.000$ \\
\hline Turdus merula & 28777 & 32242 & MF & $25.000-50.000$ \\
\hline Columba livia & 19931 & 26363 & MF & $12.500-25.000$ \\
\hline Fringilla coelebs & 13340 & 16107 & MF & $10.000-15.000$ \\
\hline Sylvia atricapilla & 12162 & 14077 & MF & $10.000-15.000$ \\
\hline Erithacus rubecula & 9356 & 11342 & MF & $7.500-12.500$ \\
\hline Parus caeruleus & 7755 & 9622 & MF & $5.000-10.000$ \\
\hline Sylvia melanocephala & 5538 & 7078 & MF & $4.000-7.500$ \\
\hline Apus unicolor $\neq$ & ¿? & ¿? & - & $5.000-12.500$ \\
\hline Pyrrhocorax pyrrhocorax & 5351 & 9114 & $\mathrm{~F}$ & $2.500-10.000$ \\
\hline Columba junoniae & 4796 & 6234 & MF & $3.000-6.000$ \\
\hline Columba bolii & 4334 & 5403 & MF & $3.000-6.000$ \\
\hline Anthus berthelotii & 4048 & 5154 & MF & $2.500-5.000$ \\
\hline Carduelis cannabina & 3003 & 5903 & $\mathrm{~F}$ & $1.000-5.000$ \\
\hline Streptopelia decaocto & 1957 & 3510 & $\mathrm{~F}$ & $1.000-5.000$ \\
\hline Streptopelia turtur & 1778 & 2511 & $\mathrm{~F}$ & $1.000-2.500$ \\
\hline Falco tinnunculus & 1390 & 1696 & MF & $1.000-2.000$ \\
\hline Motacilla cinerea & 1107 & 1720 & $\mathrm{~F}$ & $500-2.000$ \\
\hline Alectoris barbara & 922 & 1388 & $\mathrm{~F}$ & $500-2.000$ \\
\hline Sylvia conspicillata & 873 & 1419 & $\mathrm{~F}$ & $500-2.000$ \\
\hline Passer hispaniolensis & 165 & 777 & PF & $250-1.000$ \\
\hline Scolopax rusticola & 166 & 297 & $\mathrm{PF}$ & $100-500$ \\
\hline Corvus corax & 151 & 246 & $\mathrm{~F}$ & $50-250$ \\
\hline Buteo buteo & 127 & 194 & $\mathrm{~F}$ & $50-250$ \\
\hline Accipiter nisus & 110 & 220 & $\mathrm{~F}$ & $50-250$ \\
\hline Coturnix coturnix & 75 & 245 & $\mathrm{PF}$ & $<250$ \\
\hline Burhinus oedicnemus & 66 & 228 & $\mathrm{PF}$ & $<250$ \\
\hline Falco pelegrinoides & 7 & 44 & PF & $<25$ \\
\hline Carduelis carduelis* & ¿? & ¿? & - & $<50$ \\
\hline Petronia petronia* & ¿? & ¿? & - & $<50$ \\
\hline Miliaria calandra* & ¿? & ¿? & - & $<50$ \\
\hline Upupa epops* & ¿? & i? & - & $<25$ \\
\hline
\end{tabular}

*: imposibilidad de estima cuantitativa certera por trabajar con abundancias relativas (aves / km) en vez de con densidades absolutas $\left(\right.$ aves $\left./ \mathrm{km}^{2}\right)$; se proporciona el intervalo más plausible en función de las abundancias relativas obtenidas.

*: especies no detectadas, pero registradas en la literatura como reproductoras en 1990-2000.

versal permite identificar las preferencias de hábitat de cada especie, los ambientes en los que alcanzan sus máximos ecológicos, datar sus niveles máximos de densidad y estimar su valencia ecológica medida por la amplitud de hábitat. La Tabla 9 cuantifica la amplitud de hábitat de cada especie, tanto en los once ambientes distinguidos $(\mathrm{AH})$, como solamente en aquellos naturales no antropizados (AHnat).
Hay especies que alcanzan elevadas densidades en ambientes concretos, aunque no tienen elevados efectivos poblacionales en toda la isla de La Palma. Este es el caso de Anthus berthelotii que presenta elevadas densidades en los matorrales montanos, o las palomas Columba junoniae y Columba bolii que tienen elevadas densidades en la laurisilva. Las especies con efectivos poblacionales más reducidos 


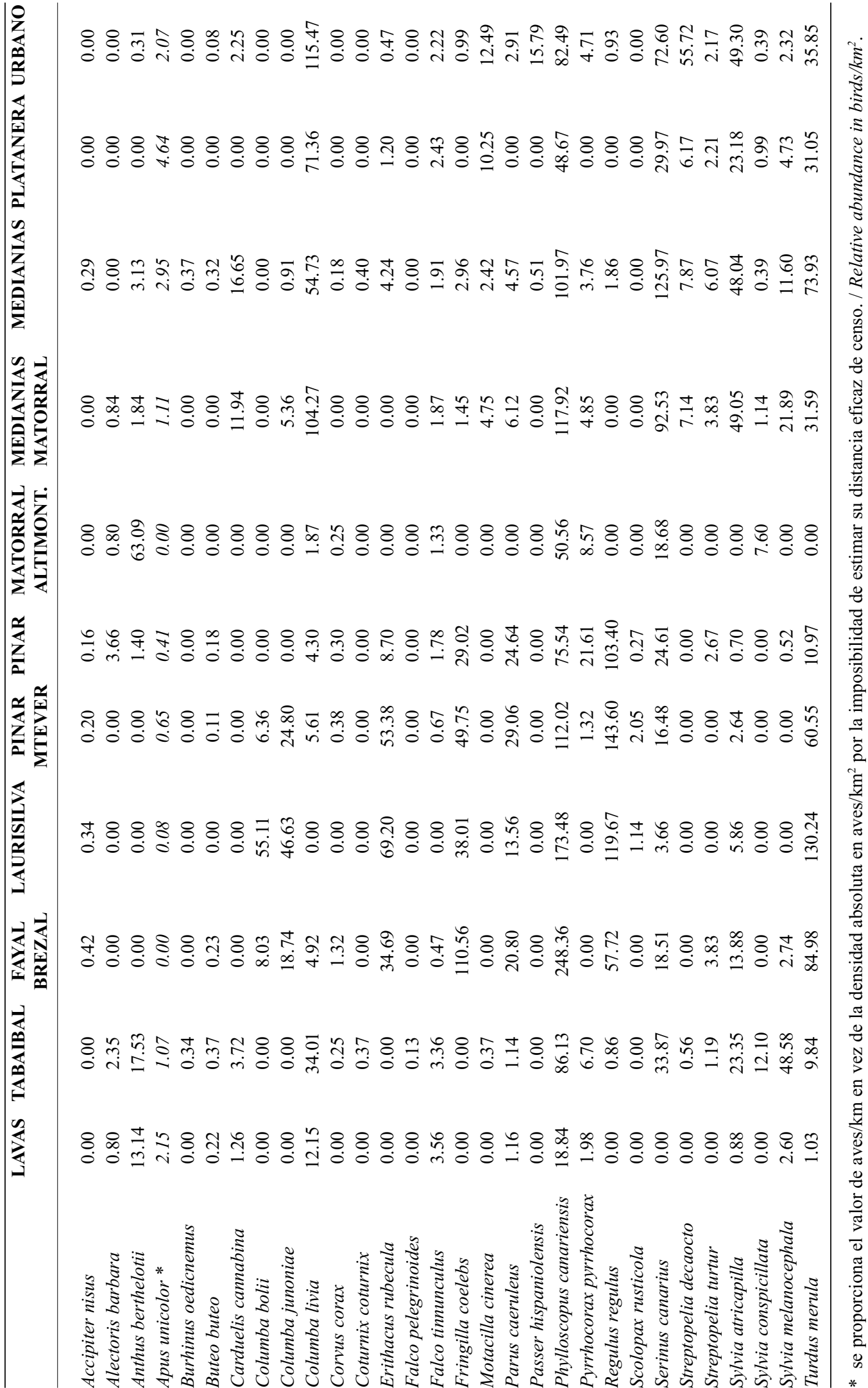


Tabla 9.- Amplitud de hábitat en las once formaciones ambientales distinguidas en La Palma (AH) y en las siete naturales (AHnat) con muy poco impacto humano (excluyendo los dos tipos de medianías, las plataneras y las áreas urbanas).

Table 9.- Habitat breadth of terrestrial bird species of La Palma island in eleven environmental units (AH) and in seven natural units with a low human influence (disregarding agricultural and urban areas; AHnat)

\begin{tabular}{lcc} 
& & \\
\hline & AH & AHnat \\
\hline Accipiter nisus & 0.41 & 0.50 \\
Alectoris barbara & 0.31 & 0.41 \\
Anthus berthelotii & 0.20 & 0.29 \\
Apus unicolor & 0.49 & 0.43 \\
Burhinus oedicnemus & 0.18 & 0.14 \\
Buteo buteo & 0.53 & 0.63 \\
Carduelis cannabina & 0.26 & 0.23 \\
Columba bolii & 0.14 & 0.22 \\
Columba junoniae & 0.27 & 0.37 \\
Columba livia & 0.45 & 0.41 \\
Corvus corax & 0.31 & 0.42 \\
Coturnix coturnix & 0.18 & 0.14 \\
Erithacus rubecula & 0.30 & 0.44 \\
Falco pelegrinoides & 0.09 & 0.14 \\
Falco tinnunculus & 0.73 & 0.60 \\
Fringilla coelebs & 0.29 & 0.43 \\
Motacilla cinerea & 0.29 & 0.14 \\
Parus caeruleus & 0.46 & 0.56 \\
Passer hispaniolensis & 0.10 & - \\
Phylloscopus canariensis & 0.74 & 0.69 \\
Pyrrhocorax pyrrhocorax & 0.40 & 0.39 \\
Regulus regulus & 0.34 & 0.53 \\
Scolopax rusticola & 0.20 & 0.31 \\
Serinus canarius & 0.52 & 0.70 \\
Streptopelia decaocto & 0.17 & 0.14 \\
Streptopelia turtur & 0.52 & 0.36 \\
Sylvia atricapilla & 0.51 & 0.41 \\
Sylvia conspicillata & 0.22 & 0.27 \\
Sylvia melanocephala & 0.27 & 0.18 \\
Turdus merula & 0.55 & 0.45 \\
\hline & & \\
& & \\
& & \\
& &
\end{tabular}

en toda la isla de La Palma ( $<250$ individuos), y que además son muy raras incluso en aquellos ambientes donde alcanzan sus máximos ecológicos (menos de 10 aves $/ \mathrm{km}^{2}$ ), son Accipiter nisus, Alectoris barbara, Burhinus oedicnemus, Buteo buteo, Corvus corax, Coturnix coturnix y Scolopax rusticola. Falco [peregrinus] pelegrinoides, Upupa epops, Carduelis carduelis, Petronia petronia y Miliaria calandra mantienen poblaciones críticas que, de existir aun, serían inferiores a los 50 individuos (con casi toda certeza de menos de 25 parejas reproductoras; en el caso de Falco [peregrinus] pelegrinoides y Upupa epops, es altamente probable que sus efectivos poblacionales sean inferiores a 12 parejas reproductoras).

Las especies que relativamente tienen menores densidades tienen en medios antropizados son Alectoris barbara, Anthus berthelotii, Columba bolii, Columba junoniae, Corvus corax, Erithacus rubecula, Falco [peregrinus] pelegrinoides, Fringilla coelebs palmae, Parus caeruleus palmensis, Pyrrhocorax pyrrhocorax, Regulus regulus ellenthalerae, Scolopax rusticola y Sylvia conspicillata. De ellas, las que tienen menor amplitud de hábitat (i.e., son más especializadas en sus preferencias de hábitat) son Anthus berthelotii, Columba bolii, Falco [peregrinus] pelegrinoides, Scolopax rusticola y Sylvia conspicillata. Otras especies bastante especializadas en sus preferencias de hábitat, pero que tienen el grueso de sus efectivos en medios antropizados, son Burhinus oedicnemus, Carduelis cannabina, Coturnix coturnix, Passer hispaniolensis y Streptopelia decaocto.

\section{REVISIÓN DEL ESTATUS DE CONSERVACIÓN EN LA PALMA}

A continuación se resumen los principales patrones de rareza ecológica y efectivos poblacionales de la avifauna terrestre reproductora de la isla de La Palma, teniendo en cuenta las tablas anteriormente presentadas, y poniendo especial énfasis en aquellas especies que tienen un nivel más crítico en la isla en función de sus efectivos poblacionales, amplitudes de hábitat, restricción a medios naturales no antropizados, y densidades en los ambientes donde alcanzan sus máximos ecológicos.

Upupa epops, Carduelis carduelis, Miliaria calandra y Petronia petronia no se han detectado en los muestreos estandarizados ni en otras prospecciones por la isla $(1.400 \mathrm{~km}$ recorridos en coche con luz diurna y 45 horas de recorridos a pié por diferentes ambientes). No han sido observadas en localidades donde previamente se había constatado su presencia según la información recopilada por Martín y Lorenzo (2001). No son ni especies ni subespecies endémicas de Canarias. A juzgar por los datos cualitativos previamente existentes (Martín y Lorenzo, 2001) han sufrido una fortísima regresión en los últimos 50 años, y la práctica total desaparición de muchas de sus localidades conocidas en los últimos 15 años. De existir aun, serían escasísimas (menos de 0,5 aves $/ \mathrm{km}^{2}$ en sus hábitats preferidos) y tendrían tamaños de población inferior a 25 parejas reproductoras. En La Palma son indiscutiblemente mucho más escasas que en otras islas occidentales, en las cuales están principalmente vinculadas a formaciones fuertemente antropizadas (Martín y 
Lorenzo, 2001; Carrascal y Palomino, 2005). El abandono de la agricultura tradicional (que ha conllevado el crecimiento de formaciones arbustivas y herbáceas muy densas), el establecimiento de monocultivos (principalmente plátano y tomate) y el uso intensivo de pesticidas, en conjunción con los modelos de urbanismo hoy día implantados, ha conducido a su virtual extinción en los ambientes fuertemente antropizados en los cuales a mediados del siglo XX eran abundantes.

Falco [peregrinus] pelegrinoides es muy escaso, estando relegado a zonas bajas cercanas a la costa, muy despobladas y con abundancia de Paloma Bravía. Aparente recuperación poblacional en los últimos 15 años (Martín y Lorenzo, 2001; Palacios, 2004).

Burhinus oedicnemus distinctus es una subespecie endémica de las islas occidentales Canarias. Muy escasa en La Palma, donde su población muy posiblemente sea de unos 70 individuos. Este pequeño tamaño poblacional, junto con los escasos efectivos presentes en otras islas occidentales (Delgado y Naranjo, 2001; Carrascal y Palomino, 2005), caracteriza el nivel crítico de sus poblaciones. Su gran rareza viene motivada tanto por la escasez de áreas adecuadas para la especie en la isla, como por su reducidísima densidad en aquellos lugares donde es más abundante $(0,3-0,4$ aves $/ \mathrm{km}^{2}$ en zonas bajas de tabaibales y medianías con cultivos y casas dispersas). En La Palma presenta una densidad máxima similar a la medida en Tenerife (Carrascal y Palomino, 2005), pero muy inferior a las medidas en Fuerteventura $(3,4$ aves $\left./ \mathrm{km}^{2}\right)$ y Lanzarote $\left(10-17\right.$ aves $/ \mathrm{km}^{2}$; Carrascal y Alonso, 2005). La mayoría de sus efectivos en La Palma se establecen en áreas antropizadas dominadas por pequeños cultivos tradicionales y casas dispersas, lo cual es consistente con sus preferencias ambientales en las islas orientales en las que manifiesta unas marcadas preferencias por áreas cercanas a pueblos con elevadas coberturas de terófitos ruderales (Palomino, 2006). Considerando la vegetación potencial de La Palma y sus preferencias de hábitat, la especie nunca debió ser abundante en la isla, como lo sugiere la literatura disponible desde mediados del siglo XIX (ver revisión en Martín y Lorenzo, 2001). El abandono de la agricultura tradicional en muchas zonas bajas y de medianía, la recuperación subsiguiente de densos tabaibales y matorrales de brezo y retama, y el establecimiento de monocultivos de plátano y tomate en las áreas de menor altitud y pendiente del terreno, con toda seguridad están limitando que la especie pueda aumentar su población en La Palma. No es posible determinar, con la mínima certeza, si sus efectivos han sufrido regresión en los últimos 50 y 15 años.

Coturnix coturnix es una especie muy escasa que se distribuye en La Palma principalmente por algunos tabaibales con gran cobertura de herbáceas y medianías con menor cobertura de matorral y más presencia de pastizales llanos. En La Palma es más escasa que en Tenerife (6 aves $/ \mathrm{km}^{2}$; Carrascal y Palomino, 2005), y mucho más que en Lanzarote $\left(148\right.$ aves $\left./ \mathrm{km}^{2}\right)$ y Fuerteventura (20 aves $/ \mathrm{km}^{2}$; Carrascal y Alonso, 2005), aunque ocupa similares formaciones ambientales dominadas por densos herbazales con poca pendiente. La gran mayoría de sus efectivos en La Palma se establecen en áreas antropizadas dominadas por pequeños cultivos tradicionales y casas dispersas. Fortísima regresión en los últimos 50 años y práctica total desaparición de muchas localidades donde era común hace 15 años, a juzgar por los datos obtenidos en este estudio y la literatura revisada por Martín y Lorenzo (2001).

Buteo buteo insularum es una subespecie endémica de Canarias, muy escasa en La Palma (menos de 0,5 aves $/ \mathrm{km}^{2}$ en sus hábitats preferidos) y con tamaño de población inferior a 250 individuos. Esta escasa abundancia tiene bases ecológicas considerando la relación alométrica existente entre tamaño corporal - área de campeo - densidad (Gaston y Blackburn, 2000), aunque su densidad ecológica máxima medida en La Palma es inferior a la datada en Tenerife $\left(1,1\right.$ aves $/ \mathrm{km}^{2}$; Carrascal y Palomino, 2006), y sustancialmente menor que la medida en áreas continentales del suroeste del Paleártico Occidental de características ambientales similares (Cantabria, Asturias y País Vasco en la Península Ibérica; Martí y del Moral, 2003). Principalmente está presente en formaciones ambientales naturales no sometidas a fuerte riesgo de degradación. La tendencia poblacional en los últimos 50 y 15 años es desconocida, aunque no es probable que haya sufrido una marcada regresión.

Accipiter nisus granti es una subespecie endémica muy escasa (densidad máxima menor de 0,5 aves $/ \mathrm{km}^{2}$ ) que sin embargo ocupa todas las áreas forestales y las medianías aledañas dedicadas a la agricultura y con urbanismo muy disperso. Tiene una amplitud de hábitat media-alta. Sus mayores efectivos se concentran en el monteverde, un ambiente en recuperación no sometido a fuertes alteraciones o peligros inminentes. No obstante, sólo se estima un promedio de unos 125 individuos en La Palma, tamaño poblacional bastante escaso para ser una subespecie endémica, de talla corporal mediana, con elevada disponibilidad de alimento (alta densidad de Mirlo en toda la isla y en los 
ambientes preferidos por el Gavilán) y con escaso efectivos en otras islas occidentales (Martín y Lorenzo, 2001). Globalmente tiene una densidad superior a la registrada en Tenerife, pero inferior a la que tiene en El Hierro (Carrascal y Palomino, 2005; Carrascal, datos inéditos). Es posible que el Gavilán haya aumentado sus efectivos por todo el norte de la isla como consecuencia del abandono de la agricultura tradicional y la recuperación del fayal-brezal que mantiene elevadísimas densidades de una de sus presas favoritas (Mirlo; Martín y Lorenzo, 2001).

Corvus corax tiene escasos efectivos en $\mathrm{La}$ Palma (estima media de 150 aves y máximo de 250) y alcanza densidades ecológicas máximas bajas (máximo de 1,3 aves $/ \mathrm{km}^{2}$ ), aunque ocupa una considerable variedad de formaciones ambientales. Manifiesta una gran amplitud de distribución altitudinal, observándose desde casi nivel del mar hasta las máximas altitudes de la isla. Su abundancia en los ambientes preferidos es considerablemente mayor que la medida en Tenerife (Carrascal y Palomino, 2005), similar a la registrada en Fuerteventura $\left(1,4\right.$ aves $\left./ \mathrm{km}^{2}\right)$, pero mucho menor que la observada en El Hierro ( 7 aves $/ \mathrm{km}^{2}$; Nogales 1994). Sólo una pequeña proporción de sus efectivos se establecen o explotan ambientes antropizados (medianías con uso tradicional del suelo y poblamiento humano muy disperso). No se puede establecer con certidumbre su tendencia poblacional en los últimos 50 y 15 años, aunque es probable que haya sido estable, o en ligero aumento (Nogales -en Martín y Lorenzo, 2001- propone en 1992 un máximo de 50 parejas reproductoras).

Scolopax rusticola, Passer hispaniolensis, Sylvia conspicillata orbitalis y Alectoris barbara tienen escasos efectivos poblacionales en La Palma (de 100 a 1.000 individuos; ver Tabla 7). Sus amplitudes de hábitat son reducidas y, salvo en el caso de Passer hispaniolensis, están virtualmente ausente de formaciones ambientales antropizadas. Sus densidades máximas ecológicas son bajas, no superando las 20 aves $/ \mathrm{km}^{2}$. Las abundancias medias y máximas de Scolopax rusticola, Passer hispaniolensis, y Alectoris barbara son considerablemente menores que las registradas en Tenerife (Carrascal y Palomino, 2005). Sylvia conspicillata orbitalis es más densa en La Palma que en Tenerife en hábitats naturales equivalentes (Carrascal y Palomino, 2005), aunque en Tenerife es mucho más abundante en ambientes antropizados que en La Palma. No se puede establecer con certeza sus tendencias poblacionales en los últimos 50 y 15 años con la información disponible, aunque es probable que sus efectivos se hayan mantenido estables a lo largo de los últimos 15 años (Scolopax rusticola y Sylvia conspicillata orbitalis) o hayan aumentado ligeramente (Passer hispaniolensis y Alectoris barbara).

El resto de las especies tienen efectivos poblacionales en La Palma superiores a 1.000 individuos, considerándose que son suficientes para mantener poblaciones estables. Motacilla cinerea canariensis y Streptopelia decaocto están mayoritariamente vinculadas a ambientes fuertemente antropizados y en expansión (como núcleos urbanos y plataneras), donde alcanzan densidades máximas de más de 10 aves $/ \mathrm{km}^{2}$. Buena parte de las poblaciones de Carduelis cannabina y Streptopelia turtur están asentadas en medios antropizados, alcanzando densidades de más de 6 aves $/ \mathrm{km}^{2}$ en medianías dedicadas a la agricultura tradicional y con poblamiento humano disperso. Sus densidades son menores en las áreas de medianía abandonadas con marcada regeneración de la vegetación nativa, que en aquellas en activo que mantienen el uso tradicional (compárense las densidades en medianíasmatorral vs. medianías en la Tabla 8). A juzgar por los datos de este trabajo y la informacón recogida en Martín y Lorenzo (2001) posiblemente hayan sufrido una regresión en los últimos 15 años como consecuencia del abandono de la agricultura tradicional. No obstante, sus efectivos son aun numerosos en la isla.

De las especies restantes, las endémicas propias de masas forestales autóctonas (Columba bolii, Columba junoniae, Parus caeruleus palmensis, Regulus regulus ellenthalerae, Fringilla coelebs palmae) tienen tamaños poblacionales que superan con toda seguridad los 3.000 individuos, alcanzan elevadas densidades máximas en sus ambientes preferidos (más de 25 aves $/ \mathrm{km}^{2}$ ) y están restringidos a formaciones vegetales sobre las que no pesan problemas de conservación graves o inminentes. No se constata para ellas tendencias demográficas negativas en los últimos 15 años a la luz de la literatura existente.

Las otras cuatro especies endémicas de Canarias no vinculadas a ambientes forestales (Falco tinnunculus canariensis, Apus unicolor, Anthus berthelotii, Serinus canarius) tienen tamaños poblacionales medios superiores a los 1.500 individuos. De ellas, Serinus canarius y Apus unicolor tienen la mayoría de sus efectivos en ambientes antropizados, muestran elevadas amplitudes de hábitat, y alcanzan localmente abundancias muy altas. Por el contrario, Anthus berthelotii tiene una amlitud de hábitat reducida, menos de una décima parte de sus efectivos se asientan en ambientes antropizados, y su 
máximo ecológico está restringido a un medio de muy reducida extensión en la isla (matorrales altimontanos; 63 aves $/ \mathrm{km}^{2}$ ). Falco tinnunculus canariensis, aunque tiene una densidad baja (máximos de 2-3 aves $/ \mathrm{km}^{2}$ ), muestra una elevada amplitud de hábitat, ocupando todas las formaciones prospectadas salvo el monteverde (ausente solamente de la laurisilva). No se constata para todas ellas tendencias demográficas negativas en los últimos 15 años a la luz de la literatura existente.

Pyrrhocorax pyrrhocorax es la única especie de aves terrestres canarias restringida a la isla de La Palma. Sus efectivos poblacionales son elevados (promedio de 5.300 individuos, y un mínimo de 2.400 aves). A pesar de que se ha señalado una regresión desde la mitad hasta finales del siglo XX (Martín y Lorenzo, 2001; País, 2005), es muy posible que esta tendencia demográfica negativa se haya ralentizado o desaparecido en los últimos 15 años a la luz de los pocos datos disponibles. Está presente con elevadas densidades en pinares $(22$ aves $/ \mathrm{km}^{2}$; sobre todo, por encima de los $1.400 \mathrm{~m}$ de altitud) sobre los que no pesan serias amenazas de conservación, y tiene una ampliud de hábitat media. Todo ello conduce a pensar que pueda ser considerada como una especie con un estatus de conservación seguro en La Palma en la actualidad. No obstante, es incuestionable que ha debido sufrir una importante regresión en zonas urbanas y en medianías como consecuencia de los cambios en los modelos urbanísticos, y el abandono de la agricultura tradicional.

Del resto de las especies, Phylloscopus canariensis, Turdus merula cabrerae y Columba livia [domestica] tienen elevadas amplitudes de hábitat y unos tamaños de población muy grandes en toda la isla (con más de 20.000 individuos), estando presentes con densidades relativamente altas en ambientes antropizados. Las otras tres restantes (Sylvia atricapilla, Sylvia melanocephala y Erithacus rubecula) no son formas endémicas de Canarias, tienen poblaciones en La Palma superiores a 5.000 individuos y ocupan una considerable variedad de ambientes. No se constata para ellas tendencias demográficas negativas en los últimos 15 años a la luz de la literatura existente.

\section{AGRADECIMIENTOS}

Este trabajo ha sido financiado por los proyectos CGL2005-02642/BOS del Ministerio de Educación y Ciencia y "Fundamentos ecologicos y biogeograficos de la rareza de la avifauna terrestre canaria" financiado por la Consejería de Medio Ambiente del Gobierno de Canarias.

\section{Referencias}

ABDI, H., 2007. Partial least square regression (PLS regression). In: N. J. Salkind (ed.). Encyclopedia of Measurement and Statistics. Sage. Thousands Oaks. $1022 \mathrm{pp}$.

ANÓNIMO, 1980. Atlas Básico de Canarias. Interinsular Canaria, S. A. Santa Cruz de Tenerife. 80 pp.

Baker, A. J., Dennison, M. D., Lynch, A. \& Legrand, G., 1990. Genetic-divergence in peripherally isolated populations of chaffinches in the atlantic islands. Evolution, 44: 981-999.

BibBy, C. J., Burgess, N. D., Hill, D. A., \& Mustoe, S. H., 2000. Bird Census Techniques, 2nd edition. Academic Press. London. 302 pp.

Buckland, S. T., Anderson, D. R., Burnham, K. P., LAAKe, J. L., Borchers, D. L. \& ThOMAS, L., 2001. Introduction to distance sampling, $1^{\text {th }}$ edition. Oxford University Press Oxford. 448 pp.

Buckland, S. T., Anderson, D. R., Burnham, K. P., LAAKe, J. L., Borchers, D. L. \& ThOMAs, L., 2004. Advanced distance sampling, $1^{\text {th }}$ edition. Oxford University Press. Oxford. 434 pp.

Carrascal, L. M. \& Alonso, C. L., 2005. Censo de aves estepáricas en las islas orientales del Archipiélago Canario. Programa de seguimiento y planificación de especies amenazadas de Canarias "CENTINELA". Consejería de Medio Ambiente, Gobierno de Canarias. Tenerife. 100 pp.

Carrascal, L. M. \& Palomino, D., 2002. Atributos insulares determinantes del número de especies por isla en el archipiélago de las Canarias y Selvagem. Ardeola, 49: 211-221.

Carrascal, L. M. \& Palomino, D., 2005. Preferencias de hábitat, densidad y diversidad de las comunidades de aves en Tenerife (islas Canarias). Animal Biodiversity \& Conservation, 28: 101-119.

Carrascal, L. M., Seoane, J., Palomino, D. \& Alonso, C. L., 2006. Preferencias de hábitat, estima y tendencias poblacionales de la Avutarda Hubara (Chlamydotis undulata) en Lanzarote y La Graciosa (Islas Canarias). Ardeola, 53: 251-269.

Carrascal, L. M., Seonne, J., Palomino, D. \& Alonso, C. L., 2007. El Corredor Sahariano en España. I Censo Nacional (2005-2006). Monografía $\mathrm{n}^{\circ} 14$. SEO/BirdLife. Madrid. $58 \mathrm{pp}$.

Delgado, G. \& Naranjo, J. J. 2001. Conservación de aves esteparias de Gran Canaria y Tenerife. Revista de Medio Ambiente. http://www.gobcan.es/cmayot/ centrodocumentacion/publicaciones/revista/separatas/avesteparias/index.html.

Dietzen, C., García-del-Rey, E., Castro, G. D. \& WinK, M., 2008. Phylogeography of the blue tit (Parus teneriffae-group) on the Canary Islands based on mitochondrial DNA sequence data and morphometrics. Journal of Ornithology, 149: 1-12. 
Donazar, J. A. Gangoso, L., Forero, M. G. \& Juste, J., 2005 Presence, richness and extinction of birds of prey in the Mediterranean and Macaronesian islands. Journal of Biogeography, 32: 1701-1713.

Frank, I. E. \& FrIEDMAN, J. H., 1993. A statistical view of some chemometrics regression tools. Technometrics, 35: 109-135.

GÄRDENFORS, U., 2001. Classifying threatened species at national versus global levels. Trends in Ecology and Evolution, 16: 511-516.

Gärdenfors, U., Hilton-Taylor, C., Mace, G. M. \& RoDRíguEZ, J. P., 2001. The application of IUCN Red List criteria at regional levels. Conservation Biology, 15: 1206-1212.

Gaston, K. J. \& Blackburn, T., 2000. Pattern and process in macroecology. Blackwell Science. Oxford. 392 pp.

Kvist, L., Broggi, J., Illera, J. C. \& Koivula, K., 2005. Colonisation and diversification of the blue tits (Parus caeruleus teneriffae-group) in the Canary Islands. Molecular Phylogenetics \& Evolution, 34: 501-511.

LEGENDRE, P. 1993. Spatial autocorrelation: trouble or new paradigm? Ecology, 74: 1659-1673.

Lorenzo, J.A. (ed.), 2007. Atlas de las Aves Nidificantes en el Archipiélago Canario (1997-2003). DGCNSEO/BirdLife. Madrid. 520 pp.

Martí, R. \& Del Moral, J. C., 2003. Atlas de las Aves Reproductoras de España. Ministerio de Medio Ambiente-SEO/BirdLife. Madrid. 733 pp.

Martín, A. \& Lorenzo, J. A., 2001. Aves del Archipiélago Canario. Francisco Lemus. La Laguna. 519 pp.

Nogales, M., 1994. High-density and distribution patterns of a raven Corvus corax population on an oceanic island (El Hierro, Canary Islands). Journal of Avian Biology, 25: 80-84.

PACKerT, M., 2006. Song dialects as diagnostic characters - acoustic differentiation of the Canary Island Goldcrest subspecies Regulus regulus teneriffae Seebohm 1883 and R. r. ellenthalerae Packert et al. 2006 (Aves: Passeriformes: Regulidae). Zootaxa, 1325: 99-115.

Packert, M., Dietzen, C., Martens, J., Wink, M. \& KVIST, L., 2006. Radiation of Atlantic goldcrests Regulus regulus spp.: evidence of a new taxon from the Canary Islands. Journal of Avian Biology, 37: 364-380.

PAIS, F. L., 2005. Problemática conservacionista de la Chova Piquirroja, Pyrrhocorax pyrrhocorax barbarus Vaurie, 1954, en La Palma, Islas Canarias (Aves, Corvidae). Revista Estudios Generales de la Isla de La Palma, $\mathrm{n}^{\mathrm{o}}$ 1. http://www.palmensis.com/estudiosgenerales/segunda/estudios $2 . h t m$.
Palacios, C. J., 2004. Current status and distribution of birds of prey in the Canary Islands. Bird Conservation International, 14: 203-213.

Palomino, D., 2005. Caracterización y uso de hábitat de aves estepáricas en las islas orientales del archipiélago canario. Programa de seguimiento y planificación de especies amenazadas de Canarias "CENTINELA". Consejería de Medio Ambiente, Gobierno de Canarias. Tenerife. 84 pp.

Scott, J. M., Heglund, P. J., Morrison, M. L., Haufler, J. B., Raphael, M. G., Wall, W. A. \& Samson, F. B., 2002. Predicting species occurrences. Issues of scale and accuracy. Island Press. Washington. $840 \mathrm{pp}$.

STATSOFT, 2001. Statistica (data analysis software system), version 6.0.

TelleríA, J. L., 1986. Manual para el censo de los vertebrados terrestres. Raíces. Madrid. 287 pp.

Thomas, L., Buckland, S. T., Burnham, K. P., Anderson, D. R., LAAKe, J. L., Borchers, D. L. \& StrindBerG, S., 2002. Distance sampling. In: A. H. El-Shaarawi \& W. W. Piegorsch (eds.). Encyclopedia of Environmetrics. John Wiley \& Sons. Chichester: 554-552.

ToBiAs, R. D. 2003. An Introduction to Partial Least Squares Regression.

URL: http://www.ats.ucla.edu/stat/sas/library/pls.pdf.
Recibido, 13-III-2008 Aceptado, 29-VII-2008 Publicado, 29-XII-2008 


\section{Apéndice / Appendix}

En este Apéndice se presenta muy sucintamente la base lógica y matemática utilizada para calcular la distancia eficaz de censo por un procedimiento alternativo que no implica la estima de todas las distancias de detección de las aves observadas mientras se efectúan censos utilizando transectos lineales. La base matemática del procedimiento es demasiado compleja como para poder ser abordada en este apartado, por lo que la metodología propuesta será objeto de un artículo específico (Carrascal, Polo y Seoane, en preparación).

Se entiende por distancia eficaz de censo aquella dentro de la cual se hubiesen observado todas las aves realmente presentes de no haber existido pérdida de detectabilidad con la distancia. Es decir, la que define cuál es la superficie del total prospectado en la que se puede asumir que se detectaron todos los individuos. Por tanto, identificar esta distancia para una especie dada implica disponer de una medida precisa de su densidad (aves/superficie).

La Figura Ap1 muestra con cuatro distribuciones de detectabilidad diferentes la aproximación utilizada. En un ambiente homogéneo (e.g., bosque de pinos) el número de aves observadas disminuye con la distancia a la línea del transecto (véanse las curvas indicativas de la probabilidad de detección). Más allá de la distancia máxima de detección (Dmax) ningún individuo es observado. Al realizar los transectos, el observador sólo estima una distancia umbral fija d (por ejemplo, $25 \mathrm{~m}$ para el caso de la mayoría de las especies de aves). Simplemente se anotan los individuos observados a una distancia menor o igual que esa distancia $\mathbf{d}$ (conteo de los individuos incluidos en la superficie A del gráfico [4] en la Figura Ap1), y los individuos detectados a más de esa distancia (superficie C). El número total de individuos detectados quedaría representado por la superficie bajo las curvas de la Figura Ap1 (superficies A y C en el gráfico [4]). Por tanto, entre el observador y la distancia máxima de detección existirían $\mathrm{A}+\mathrm{C}$ aves observadas y $\mathrm{B}+\mathrm{D}$ individuos no detectados. La probabilidad de detección (P) de la especie sería $(A+C) /(A+B+C+D)$. Considerando la distancia máxima de detección, la distancia eficaz de censo (DEC) sería P·Dmax.

Al realizar los transectos con una distancia umbral d se puede estimar la proporción de aves $(\mathbf{p}(\mathbf{d})=\mathrm{A} /(\mathrm{A}+\mathrm{C}))$ detectadas a una distancia menor o igual que d. Independientemente de cuál sea la función matemática que mejor describe la disminución de la detectabilidad con la distancia al observador (p.ej., seminormal, tasa de riesgo o exponencial negativa, con o sin ajustes cosenos o polinomiales; Thomas et al., 2002), se puede demostrar mediante cálculo integral que la distancia eficaz (DEC) de censo es:

$$
\text { DEC }=\mathbf{d} / \mathbf{p}(\mathbf{d})+\mathbf{t}
$$

donde $\mathbf{d}$ es la distancia umbral que define la banda de censo a ambos lados del observador ( $25 \mathrm{~m}$ en el caso de este estudio). El término $\mathbf{t}$ depende de los parámetros matemáticos de la función que describe la pérdida de la detectabilidad con la distancia.

Utilizando distintas distribuciones de detectabilidad (seminormal, tasa de riesgo o exponencial negativa), y postulando diferentes distancias máximas de detección que han oscilado entre 35 y $500 \mathrm{~m}$, se han simulado 223 especies teóricas utilizando Microsoft Excel. Para cada una de ellas se han generando 10.000 valores de distancias de detección con los que construir 223 curvas diferentes (como las cuatro de ejemplo de la Figura Ap1). A partir de los histogramas de distancias de estas curvas, se

Figura Ap1.- Ilustración gráfica del cálculo de la probabilidad de detección de una especie (P), la distancia eficaz de censo (DEC), y la proporción de los individuos detectados $(\mathbf{p}(\mathbf{d}))$ que fueron observados a una distancia menor o igual que un valor umbral d. Se representan cuatro especies hipotéticas ([1] a [4]) con cuatro funciones de distribución distintas de la disminución de la cantidad de individuos detectados con la distancia al observador. Dmax: distancia máxima de detección por encima de la cual ya no se observan más individuos. La superficie A denota el número de individuos observados a una distancia menor o igual que la umbral d, mientras que la superficie B cuantifica el número de individuos existentes que no fueron observados durante el censo dentro de esa banda de censo. Las cantidades C y D serían, respectivamente, los individuos detectados y no detectados más allá de la distancia umbral de censo d. En la esquina superior derecha se ilustra cual es la relación existente entre la distancia eficaz de censo real (DEC) y la estimada a partir de una ecuación sencilla (DEC') para 223 especies virtuales simuladas con diferentes funciones de distribución y distancias máximas de detección. En dicha relación se posicionan las cuatro especies representadas por los gráficos [1] a [4].

Figure Ap1.- Graphical illustration of the estimation of detection probability (P), efficient census strip width (DEC), and an alternative method of estimation of DEC considering the proportion of all detected individuals that were observed within a census belt of width $\mathbf{d}$ at both sides of the transect line (p(d)). Dmax: maximum distance of detection. It is shown the relationship between the theoretical value of DEC and that derived from $\mathbf{p}(\mathbf{d})(\mathbf{D E C})$ in the upper right corner of the figure. 

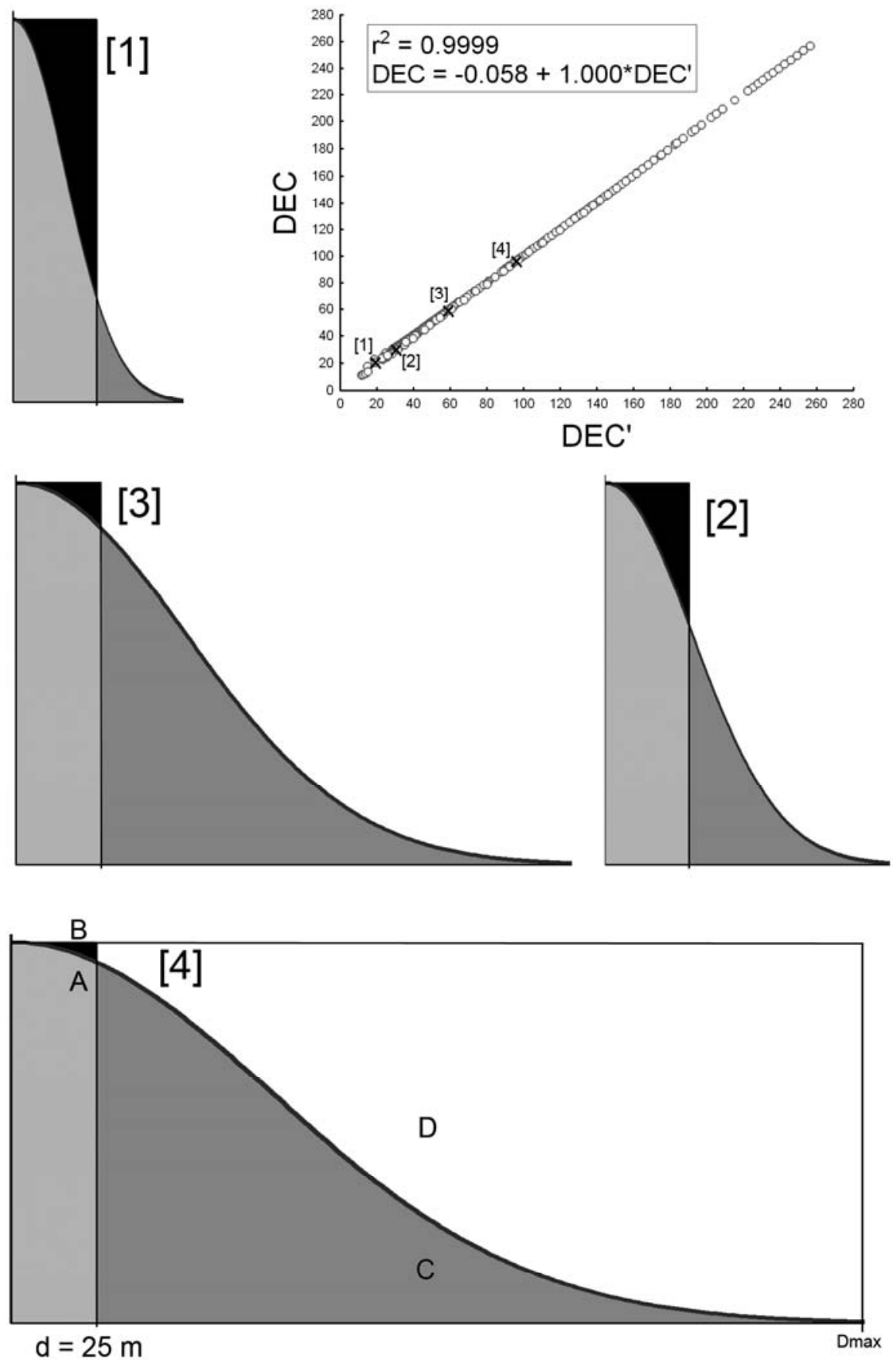

$$
\begin{array}{ll}
\mathrm{P}=(\mathrm{A}+\mathrm{C}) /(\mathrm{A}+\mathrm{B}+\mathrm{C}+\mathrm{D}) & \mathrm{p}(\mathrm{d})=\mathrm{A} /(\mathrm{A}+\mathrm{C}) \\
\mathrm{DEC}=\mathrm{Dmax} \cdot \mathrm{P} & \mathrm{DEC}=\mathrm{d} / \mathrm{p}(\mathrm{d})+t
\end{array}
$$

$$
\operatorname{Dmax} \cdot \mathrm{P}=\mathrm{d} / \mathrm{p}(\mathrm{d})+t
$$




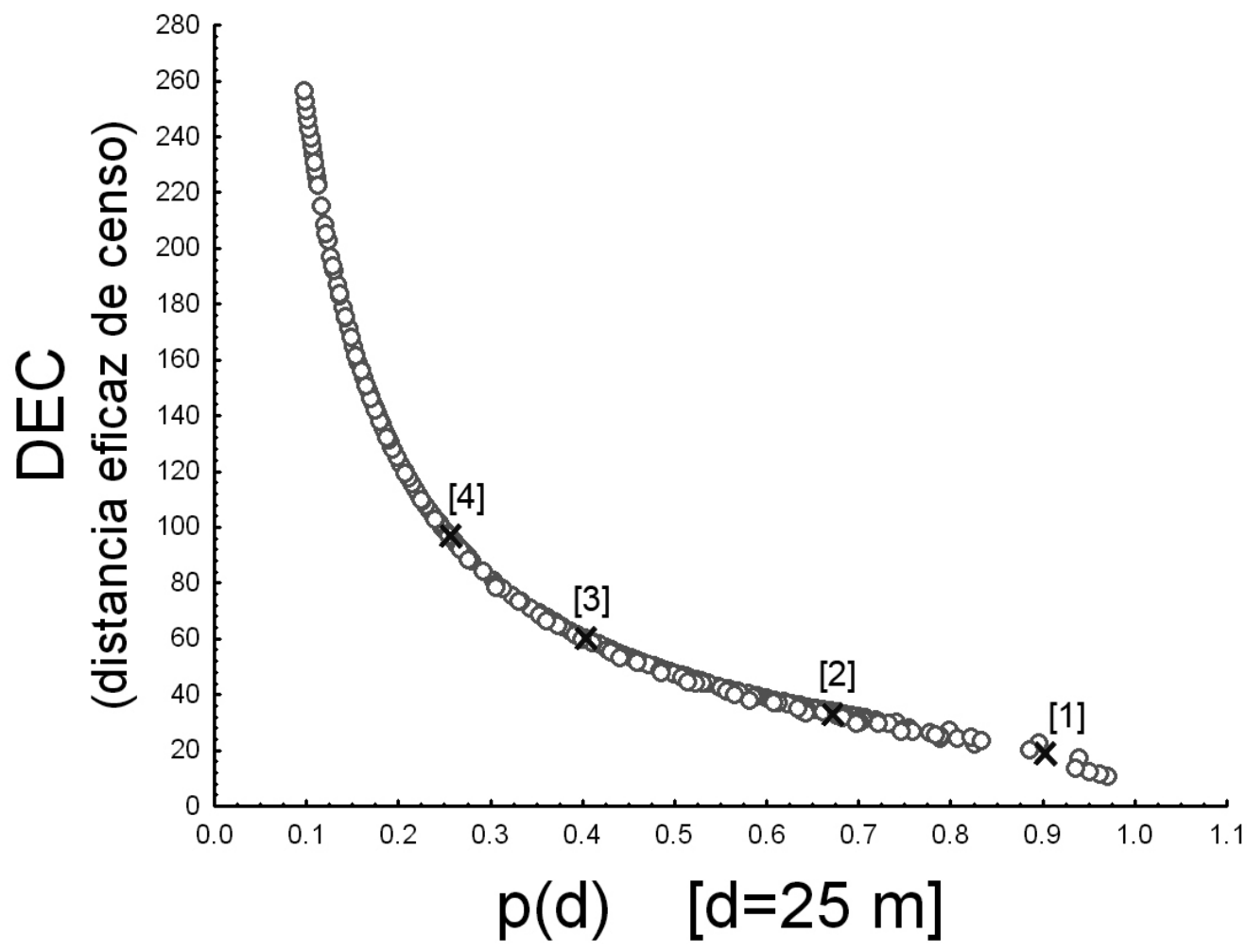

Figura Ap2.- Relación existente entre la distancia eficaz de censo (DEC) y la proporción de los individuos detectados (p(d)) que fueron observados a una distancia menor o igual que un valor umbral d. La relación se ha establecido con 223 especies virtuales simuladas con diferentes funciones de distribución y distancias máximas de detección. En dicha relación se posicionan las cuatro especies representadas por los gráficos [1] a [4] en la Figura Ap1.

Figure Ap2.- Relationship between the efficient census strip width (DEC) and the proportion of all detected individulas that were observed within a census belt $\mathbf{d}$ at both sides of the transect line $(\mathbf{p}(\mathbf{d}))$. $\mathrm{d}=25 \mathrm{~m}$ in this simulation example of 223 species of known probability of detection and maximum distance of detection. See Figure Ap1 for more details.

han calculado las superficies A, B, C y D de la Figura Ap1, la probabilidad de detección de la especie (p), la proporción $(\mathbf{p}(\mathbf{d}))$ de aves detectadas por debajo de la distancia umbral d, y la distancia eficaz de censo (DEC).

La Figura Ap2 ilustra cuál es la relación existente entre las variables p(d) y DEC. En dicha figura se señalan las posiciones que ocuparían las cuatro funciones de distribución de detectabilidad representadas en la Figura Ap1 ([1] a [4]). La relación es negativa, curvilínea y muy intensa. El modo de linealizar dicha relación es mediante la transformación inversa $(1 / \mathrm{X})$ de $\mathbf{p}(\mathbf{d})$, utilizando como constante que la multiplica la distancia umbral d; esto es, el valor $\mathbf{d} / \mathbf{p}(\mathbf{d})$. La relación entre DEC y $\mathbf{d} / \mathbf{p}(\mathbf{d})$ para la muestra de las 223 especies simuladas es $\mathrm{r}=0,9996(\mathrm{p}<<0,001)$. No obstante, dicha relación es menor cuando se utilizan las especies para las cuales la proporción de aves detectadas a una distancia menor o igual que la distancia umbral $\mathbf{d}$ es muy grande (véanse a modo de ejemplos gráficos las distribuciones [1] y [2] de la Figura Ap1 en las cuales los valores p(d) son respectivamente 0,905 y 0,678). Así, para valores de p(d) $>0,666$ la relación, aunque significativa, es menos intensa $(r=0,968, n=42$ especies simuladas, $\mathrm{p}<<0,001)$. Por este motivo, es necesario introducir el término $\mathbf{t}$ que corrige los desvíos de la linealidad entre DEC y d/p(d), especialmente para valores de $\mathbf{p}(\mathbf{d})$ mayores de 0,666 (correspondiente a especies con las que la mayoría de los contactos se dan a muy corta distancia del observador). Dicho término $\mathbf{t}$, está muy intensamente relacionado con $\mathbf{d} / \mathbf{p}(\mathbf{d})$, por lo cual para calcularlo no es necesario conocer los parámetros que definen las distintas funciones de ajuste a modelos de detectabilidad (p.ej., seminormal, 


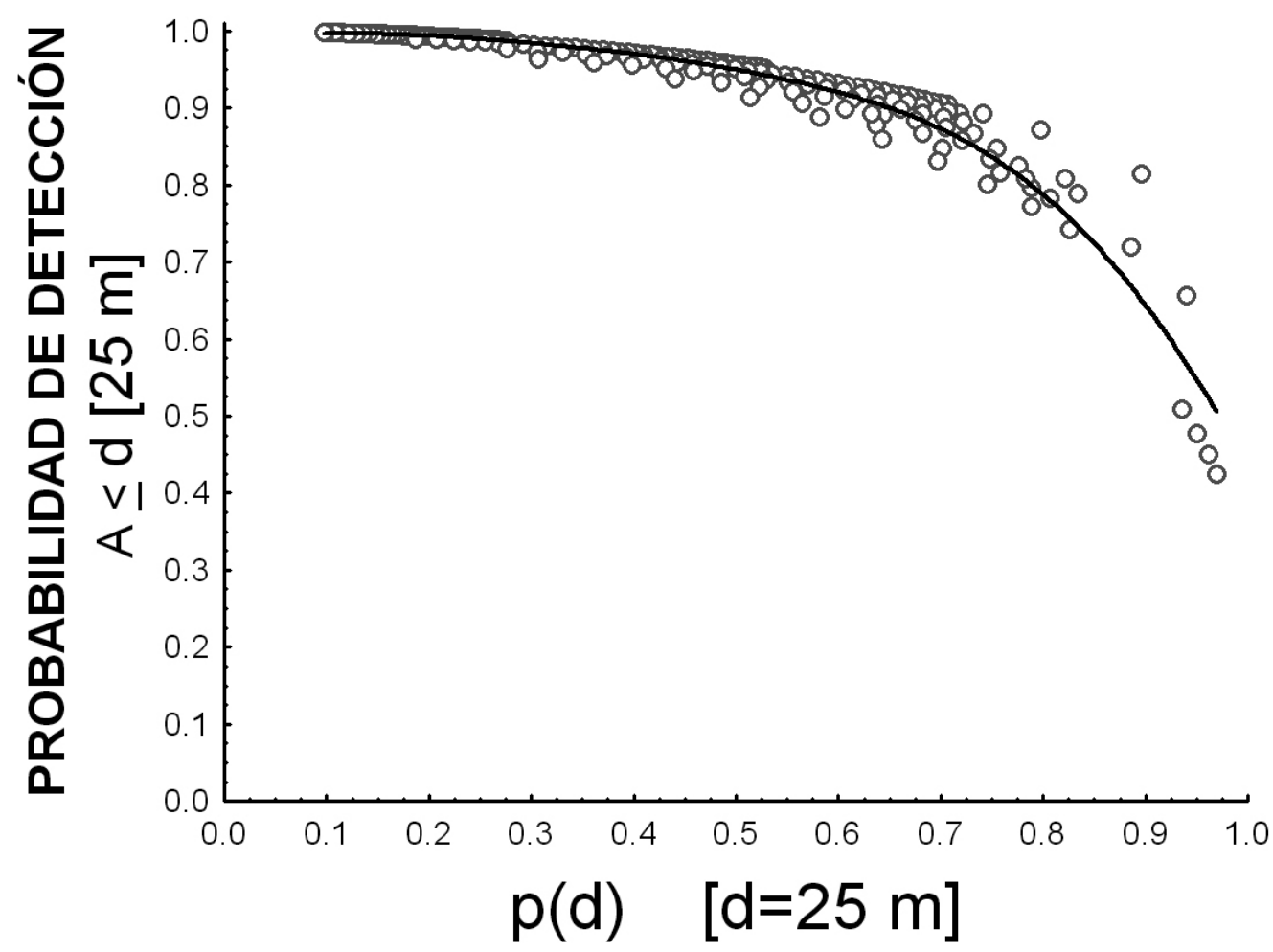

Figura Ap3. - Relación entre la proporción $(\mathbf{p}(\mathbf{d}))$ de aves observadas por debajo de la distancia umbral d, y su probabilidad de detección dentro de esas bandas.

Figure Ap3.- Relationship between the probability of detection within a census belt of distance d at both sides of the transect line (Y axis), and the proportion of all detected individulas that were observed within a census belt $\mathbf{d}$ at both sides of the transect line (p(d)). $d=25 \mathrm{~m}$ in this simulation example of 223 species of known probability of detection and maximum distance of detection.

tasa de riesgo o exponencial negativa, con o sin ajustes cosenos o polinomiales).

Utilizando las simulaciones de las 223 especies se obtiene que trabajando con transectos lineales y distancias umbral $\mathbf{d}=25 \mathrm{~m}$ a cada lado del transecto, la distancia eficaz de censo estimada (DEC') se puede calcular mediante la siguiente expresión:

$\mathbf{D E C}^{\prime}=[25 / \mathbf{p}(\mathbf{2 5})]+1 /\left(0.6861-0.1202 \cdot[25 / \mathbf{p}(\mathbf{2 5})]^{0.567}\right)$

(la segunda parte de la igualdad que se suma a [25 / $\mathbf{p ( 2 5 )}$ ] es el término $\mathbf{t}$ ).

Utilizando dicha ecuación, las distancias eficaces de censo reales (DEC) y estimadas (DEC') son virtualmente idénticas: $\mathrm{r}^{2}=0,99999 ; \mathbf{D E C}=-0,06+1,0001 \cdot$ DEC' (véase la parte superior de la Figura Ap1). Téngase en cuenta que la adición del complejo término $\mathbf{t}$ sólo contribuye muy marginalmente a perfeccionar la predicción de la distancia eficaz de censo real, incrementando la $\mathrm{r}^{2}$ sólo 0,0004 .

El intervalo de confianza de DEC en la ecuación anterior se calcula del siguiente modo a partir del intervalo de confianza de $\mathbf{p}(\mathbf{2 5})$ para una probabilidad ? dada:

$$
\mathrm{T}_{\alpha} \cdot[\mathbf{p}(\mathbf{2 5}) \cdot(1-\mathbf{p}(\mathbf{2 5}))]^{0.5} \cdot \mathrm{N}^{0.5}
$$

donde $\mathrm{T}_{\alpha}$ es el valor de la $\mathrm{T}$ de Student para N-1 grados de libertad (siendo $\mathrm{N}$ el número de contactos con individuos distintos).

El procedimiento previamente presentado también es de utilidad para estimar aproximadamente cuál es la probabilidad de detectar a las especies dentro de las bandas de censo a ambos lados del observador definidas por la distancia $\mathbf{d}$ (cociente entre las superficies $\mathrm{A} /(\mathrm{A}+\mathrm{B})$ en la Figura Ap1). La Figura Ap3 ilustra la relación entre la proporción $(\mathbf{p}(\mathbf{d}))$ de aves observadas por debajo de la 
distancia umbral d, y su probabilidad de detección dentro de esas bandas. La relación es significativa y negativa, aunque sólo explica el $95,6 \%$ de la variación observada $(\mathrm{r}=0,978, \mathrm{n}=223$ especies simuladas, $\mathrm{p}<<0,001)$. Este desvío de la absoluta certidumbre en la predicción de la probabilidad de detectar a las especies dentro de las bandas de censo a ambos lados del observador, se debe a que dicha probabilidad se ve muy influida por la forma exacta de la función de distribución que describe la disminución de la detectabilidad con la distancia al observador. La complejidad de esas funciones de distribución, y sus pequeñas variaciones a distancias cercanas al observador, no pueden ser exactamente predichas por un único y sencillo valor $\mathbf{p ( d )}$. No obstante, la relación presentada en la Figura Ap3 tiene valor heurístico postulando cuáles serían los valores más plausibles de probabilidad de detección en bandas de censo cercanas al observador.

Para las especies analizadas en este estudio, los valores de $\mathbf{p}(\mathbf{d}=\mathbf{2 5})$ oscilaron entre 0,89 para Regulus teneriffae y 0,11 de Buteo buteo. Sus distancias eficaces de censo (DEC) han oscilado entre 19 y $225 \mathrm{~m}$, respectivamente. La probabilidad de detectar a las especies dentro de las bandas de censo de $25 \mathrm{~m}$ a ambos lados del observador sería 0,65 para Regulus teneriffae y 0,99 de Buteo buteo.

Para aplicar parsimoniosamente este procedimiento, utilizando sólo la sencilla ecuación DEC' $=[25 / \mathbf{p}(25)]$, lo ideal sería efectuar un estudio piloto trabajando con diferentes bandas de censo. Teniendo en cuenta los resultados obtenidos para unos 20-25 contactos con la especie objeto de estudio se podría seleccionar aquella distancia umbral de censo $\mathbf{d}$ que arrojase valores de $\mathbf{p}(\mathbf{d})$ $<0,666$ (por ejemplo valores comprendidos entre $0,1 \mathrm{y}$ $0,5)$. Posteriormente se efectuaría el censo con bandas de recuento fijadas en esa distancia d (por ejemplo, $3 \mathrm{~m}$ para lagartijas, $25 \mathrm{~m}$ para muchas aves de tamaños menores de $500 \mathrm{~g}, 100 \mathrm{~m}$ para aves rapaces diurnas, etc). La ventaja de este procedimiento es que:
1 puede ser utilizado por un amplio conjunto de observadores que sólo están obligados a entrenarse en la estima de una sola distancia fija.

2 no es necesario estimar las distancias perpendiculares a la línea de censo de cada uno de los contactos, aspecto tremendamente difícil y de carácter 'subjetivo' en medios forestales en los que la vegetación impide la visión de los individuos o distorsiona diferencialmente la propagación del sonido. Sólo hay que determinar si los contactos quedan fuera o dentro de la banda de recuento.

3 evita el problema de inflado de algunos valores de distancia por redondeo al estimar todas las distancias de detección (e.g., gran frecuencia de distancias de $50 \mathrm{~m} \mathrm{o} 100 \mathrm{~m}$ al 'fijar' las estimas a estos valores redondeados).

4 evita tener que truncar la distribución de los datos teniendo en cuenta los contactos más lejanos y la incertidumbre de haber estimado correctamente las distancias máximas. Esto es especialmente problemático al trabajar con especies escasas de las que se han obtenido pocos contactos, y el carácter subjetivo de cuántos contactos lejanos eliminar y a partir de qué distancia. La eliminación de esos contactos lejanos puede afectar seriamente a las estimas de densidad y tamaño de población en las áreas en las que la especie es más escasa.

5 no hay que llevar a cabo prolijos cálculos matemáticos basados en el ajuste de los valores de distancia medidos a varias familias de funciones de distribución y sus variantes coseno o polinomiales (Thomas et al., 2002).

6 el procedimiento basado en la estima de $\mathrm{p}(d)$ puede ser aplicado empleando herramientas informáticas sencillas y ampliamente difundidas (e.g., hojas de cálculo) sin tener que recurrir a programas complicados con una curva de aprendizaje lenta. 